\title{
Synthesis of New Series Bis-3-Chloro- $\beta$-Lactam Derivatives from Symmetrical Bis-Schiff Bases as Effective Antimicrobial Agents with Molecular Docking Studies
}

\author{
Sangar A. Hassan ${ }^{\mathrm{a}, *}$, Media N. Abdullah ${ }^{\mathrm{b}}$, Dara M. Aziz ${ }^{\mathrm{c}}$
}

${ }^{\text {a,c }}$ College of Science, University of Raparin, Ranya, Kurdistan Region, Iraq - (sangar.ali, darachem)@uor.edu.krd

${ }^{\mathrm{b}}$ College of Science, Salahaddin University-Erbil, Kurdistan Region, Iraq - (media.abdullah@su.edu.krd)

\begin{abstract}
:
Endeavoring to find a new type of antimicrobial agents, a new sequence of bis-Schiff bases and bis-3-cholro- $\beta$-lactams are synthesized. An astonishing class of strained compounds is part of the symmetrical bis-Schiff bases that has widespread applications and building blocks for the combination of bis-3-cholro- $\beta$-lactams antibiotics. Bis-3-cholro- $\beta$-lactams are synthesized through (Staudinger) [2+2] ketene-imine cycloaddition reaction. Structures of the produced compounds are deduced by ${ }^{1} \mathrm{H},{ }^{13} \mathrm{C}-$ NMR, and FT-IR spectroscopies. All produced compounds are shown moderate to good antimicrobial activity compared to human pathogenic bacteria strains (Escherichia coli and Staphylococcus aureus), also compared to Aspergillus Niger and Trichophyton mentagrophytes fungi through the broth microdilution technique. A molecular docking study is used for showing the active sides and binding affinity of the products with the target proteins or receptors of E. coli (PDB ID: 3GI9).
\end{abstract}

KEYWORDS: Bis-Schiff bases, Bis- $\beta$-lactams, Antimicrobial activity, and Molecular docking study.

\section{INTRODUCTION}

The world's mortality rate has increased because of the increase of infectious diseases that are unswervingly related to the bacteria and fungi displaying various antibiotics confrontation. During many years, a lot of attention has been paid to the synthesis of di-imino-Schiff bases study. Schiff bases, a class of organic compounds that are characterized by $-\mathrm{N}=\mathrm{CH}$ - (imine) (Halve et al., 2006). The antibacterial activity of the bis-heterocyclic compound is proven firstly by the bis-heterocyclic character and secondly by the variation of benzene ring substitution (Shneine et al., 2017). Synthesis and characterization of Schiff bases and their corresponding heterocyclic derivatives are very important because of their biological importance. Recently abundant Schiff bases synthesized as probable bioactive core (Kajal et al., 2013, Shockravi et al., 2009), which present a significant role as an intermediate in the synthesis of some bioactive compounds such as $\beta$-lactams (Anacona and Acosta, 2006). The small molecule azomethines are explored as the conjugated linker unit for photovoltaic application (Petrus et al., 2014). Schiff bases are used as substrates in the preparation of a number of industrial and biologically active compounds via ring closure, cycloaddition and replacement reactions (AbouMelha and Faruk, 2008). New open and macrocyclic bis Schiff base were found to have moderate to strong biological activity (Essa et al., 2012). Bis-compounds are often referred to as Salen-type compounds, they are highly active against several diseases and have a potential applications as anticancer (Nworie, 2016), anticonvulsant, antitumor, antifungal, and antibacterial (Jarrahpour et al., 2007, Nazeruddin, 2016), anti-leishmanial agent (Taha et al., 2020) antitubercular and antioxidant (Khan et al., 2011), antimalaria, anti-inflammatory and corrosion inhibitor (Arshad et al., 2020, Liang et al., 2019). Azetidinone are the 2-carbonyl derivatives of
more commonly known as $\beta$ -

\footnotetext{
* Corresponding author
}

This is an open access under a CC BY-NC-SA 4.0 license (https://creativecommons.org/licenses/by-nc-sa/4.0/) lactams (Kaur et al., 2020a). Beta-lactams are one of the essential heterocycles which have saved humans from deadly infections (Kaur et al., 2018). $\beta$-lactams are widely recognized for their biological importance (Sharma et al., 2009) as antibacterial agents (Hassan, 2019), some have antimicrobial (Kaur et al., 2020b), anti-tubercular and anti-inflammatory activities (Anusha et al.). Bis-2-azetidinones have antioxidant (Shinde et al., 2015), anti-bacterial, and antifungal activities (Al-Azzawi and AlObiadi, 2016, Hassan and Aziz, 2021, N'dri et al., 2019, Pagadala et al., 2010). The continuous increase in the resistance of the bacteria against the currently existing antibiotics demanded the excessive requests for the development of a new type of compound bearing a bis-3-chloro- $\beta$-lactams pattern to overcome the challenge of bacterial resistance. For that reason, this research included the synthesis of a molecule bearing more than one $\beta$ lactam ring unit for enhancing the $\beta$-lactam antibiotic's biological activity and study their molecular docking activity.

\section{EXPERIMENTAL SECTION}

Chemical Compounds were obtained from Sigma-Aldrich, Fluka, and Merck Chemical Companies and utilized lacking any additional refinement. Melting points were measured using electrothermal melting point apparatus (Stuart, normal power 75 W, model: ST15 OSA at Raparin University) in capillaries and were uncorrected. Thin-layer chromatography (TLC) on a precoated $\mathrm{SiO}_{2}$ gel (HF254, 200 mesh) aluminum plate was utilized to test out the evolution of the reactions. TLC plates were seen by UV light (CSL-MDOCBASIC at Raparin University). The Ultrasonic cleaner was used for Sonication (DIGITAL PRO+, 40 $\mathrm{kHz}$, and a standard power $180 \mathrm{~W}$ at Raparin University). FT-IR spectra were documented on the Thermo Scientific spectrometer (Model: Nicolet iS 10 at Raparin University). ${ }^{1} \mathrm{H}$ - and ${ }^{13} \mathrm{C}-\mathrm{NMR}$ spectra were performed on a Bruckner $\left({ }^{1} \mathrm{H}: 400 \mathrm{MHz}\right.$ and ${ }^{13} \mathrm{C}$ : $100 \mathrm{MHz}$ at Tehran University/ Iran), DMSO was used as a solvent with inner standard TMS, and data were reported as the chemical shift in ppm. A microplate reader (BioTek-ELx808 at Raparin University) was used for evaluating the antimicrobial activity of the synthesized compounds. The abbreviations include 
( $\mathrm{s}=$ singlet, $\mathrm{d}=$ doublet, $\mathrm{t}=$ triplet, $\mathrm{br}=$ broad, $J=$ coupling constant, $\mathrm{Hz}=$ Hertz and $\mathrm{MP}=$ melting point).

2.1. Synthetic method for bis-Schiff bases synthesis (3a-h) An appropriate synthetic method was used with a minor modification (Hassan and Aziz, 2021). Various substituted benzaldehyde (2a-h) (2mmol) was reacted with $(1 \mathrm{mmol})$ of 4,4'-methylenedianiline (1), $10 \mathrm{~mL}$ of absolute ethanol was used as a solvent and a catalytic quantity of glacial acetic acid (3-5 drops) are used. The solution was irradiated in ultrasonic bath at $70^{\circ} \mathrm{C}$ for $(10-20)$ minutes. The achievement of the reaction was controlled by a thin layer chromatography. The solvent was removed through a rotary evaporator in order to obtain a solid product and the precipitate was purified by column chromatography using Diethyl ether: $\mathrm{MeOH}$ : Ethyl acetate $(75: 10: 15)$ as eluent.

2.1.1. $\quad N, N^{\prime}$-(methylenebis(4,1-phenylene))bis(1-phenylmethanimine) (3a):Yellowish color solid, Yield $=88 \%$, M.P $=118-119^{\circ}$ C.FT-IR $\left(\mathrm{cm}^{-1}\right): 3034$ (C-H Ar. str.), 2921, 2850 (C-H Aliph. str.), $1623\left(\mathrm{C}=\mathrm{N}_{\text {str. imine }}\right), 1577\left(\mathrm{C}=\mathrm{C}_{\text {Ar. str. }}\right) .{ }^{1} \mathrm{H}-\mathrm{NMR}$ (DMSO- $d_{6}$ ) ppm: $\delta 3.983\left(\mathrm{~s}, 2 \mathrm{H}\left(\mathrm{CH}_{2}\right)\right), 7.230-7.252(\mathrm{~d}, 4 \mathrm{H}$ $(\mathrm{H} 2, \mathrm{H} 6), J=8.8 \mathrm{~Hz}), 7.296-7.320$ (d, 4H (H3, H5), $J=9.6 \mathrm{~Hz})$, 7.496-7.517 (d, 4H (H3', H5'), J=8.4 Hz), 7.567 (t, 2H (H4'), $J=9.2 \mathrm{~Hz}, J=9.2 \mathrm{~Hz}), 7.952-7.973\left(\mathrm{~d}, 4 \mathrm{H}\left(\mathrm{H}^{\prime}{ }^{\prime}, \mathrm{H}^{\prime}\right), J=8.4\right.$ $\mathrm{Hz}), 8.610(\mathrm{~s}, 2 \mathrm{H}(\mathrm{CH}=\mathrm{N})) .{ }^{13} \mathrm{C}-\mathrm{NMR}\left(\mathrm{DMSO}-d_{6}\right) \mathrm{ppm}: \delta$ $45.73\left(1 \mathrm{C},\left(\mathrm{CH}_{2}\right)\right), 121.12(4 \mathrm{C}(\mathrm{C} 2, \mathrm{C} 6)), 128.55$ (4C (C3', $\left.\left.\mathrm{C}^{\prime}\right)\right), 128.77\left(4 \mathrm{C}\left(\mathrm{C} 2^{\prime}, \mathrm{C} 6^{\prime}\right)\right), 129.40\left(2 \mathrm{C}\left(\mathrm{C} 4^{\prime}\right)\right), 129.45(4 \mathrm{C}$ (C3, C5)), 136.05 (2C (C1')), 139.25 (2C (C4)), 149.35 (2C (C1)), $160.10(1 \mathrm{C}(\mathrm{C}=\mathrm{N}))$.

2.1.2. $\quad N, N^{\prime}$-(methylenebis(4,1-phenylene))bis(1-(4-bromophenyl)methanimine) (3b): Light red color solid, Yield $=84 \%$, M.P $=155-156^{\circ}$ C. FT-IR $\left(\mathrm{cm}^{-1}\right): 3044(\mathrm{C}-\mathrm{H}$ Ar. str.), 2923, 2853 (C-H Aliph. str. $), 1618\left(\mathrm{C}=\mathrm{N}_{\text {str. imine }}\right), 1581(\mathrm{C}=\mathrm{C}$ Ar. str.). ${ }^{1} \mathrm{H}-\mathrm{NMR}$ (DMSO- $\left.d_{6}\right)$ ppm: $\delta 3.973\left(\mathrm{~s}, 2 \mathrm{H}\left(\mathrm{CH}_{2}\right)\right)$, 6.968-6.992 (d, 4H (H2, H6), $J=9.6 \mathrm{~Hz}), 7.008-7.031(\mathrm{~d}, 4 \mathrm{H}$ (H3, H5), $J=9.2 \mathrm{~Hz}), 7.207-7.227$ (d, 4H (H3', H5'), $J=8 \mathrm{~Hz}$ ), 7.791-7.812 (d, 4H $\left.\left(\mathrm{H}^{\prime}, \mathrm{H}^{\prime}\right), J=8.4 \mathrm{~Hz}\right), 8.508(\mathrm{~s}, 2 \mathrm{H}$ $(\mathrm{CH}=\mathrm{N})) .{ }^{13} \mathrm{C}-\mathrm{NMR} \quad\left(\mathrm{DMSO}-d_{6}\right) \quad \mathrm{ppm}: \delta 44.00 \quad(1 \mathrm{C}$ $\left.\left(\mathrm{CH}_{2}\right)\right), 121.39(4 \mathrm{C}(\mathrm{C} 2, \mathrm{C} 6)), 125.60\left(2 \mathrm{C}\left(\mathrm{C}^{\prime}\right)\right), 128.55(4 \mathrm{C}$ $\left.\left(\mathrm{C} 2^{\prime}, \mathrm{C} 6^{\prime}\right)\right), 130.79(4 \mathrm{C}(\mathrm{C} 3, \mathrm{C} 5)), 132.04\left(4 \mathrm{C}\left(\mathrm{C}^{\prime}, \mathrm{C}^{\prime}\right)\right)$, $137.74\left(2 \mathrm{C}\left(\mathrm{C}^{\prime}\right)\right), 140.35(2 \mathrm{C}(\mathrm{C} 4)), 151.06(2 \mathrm{C}(\mathrm{C} 1))$, $159.74(1 \mathrm{C}(\underline{\mathrm{C}}=\mathrm{N}))$.

2.1.3. 4,4 '-((methylenebis(4,1-phenylene))bis(azaneylylidene))bis(methaneylyliden -e))diphenol (3c): Light yellow color solid, Yield $=86 \%$, M.P $=243-244^{\circ} \mathrm{C}$. FT-IR $\left(\mathrm{cm}^{-1}\right)$ : 3368 (OH $\left.{ }_{\text {str. }}\right), 3055$ (C-H Ar. str.), 2932, 2861 (C-H Aliph. str.), $1615\left(\mathrm{C}=\mathrm{N}_{\text {str. imine }}\right), 1591(\mathrm{C}=\mathrm{C}$ Ar. str. $) .{ }^{1} \mathrm{H}-\mathrm{NMR}\left(\mathrm{DMSO}-d_{6}\right)$ ppm: $\delta 3.945\left(\mathrm{~s}, 2 \mathrm{H}\left(\mathrm{CH}_{2}\right)\right), 6.881-6.901\left(\mathrm{~d}, 4 \mathrm{H}\left(\mathrm{H}^{\prime}, \mathrm{H} 5^{\prime}\right)\right.$, $J=8 \mathrm{~Hz}$,), 7.139-7.160 (d, 4H (H2, H6), $J=8.4 \mathrm{~Hz}), 7.239$ $7.259(\mathrm{~d}, 4 \mathrm{H}(\mathrm{H} 3, \mathrm{H} 5), J=8 \mathrm{~Hz}), 7.754-7.776(\mathrm{~d}, 4 \mathrm{H}(\mathrm{H} 2$, $\left.\left.\mathrm{H}^{\prime}\right), J=8.8 \mathrm{~Hz}\right), 8.443(\mathrm{~s}, 2 \mathrm{H}(\mathrm{CH}=\mathrm{N})), 10.121(\mathrm{br}, \mathrm{s}, 2 \mathrm{H}$ $(\mathrm{OH})) .{ }^{13} \mathrm{C}-\mathrm{NMR}$ (DMSO-d 6 ppm: $\delta 44.44\left(1 \mathrm{C}\left(\mathrm{CH}_{2}\right)\right)$, 115.82 (4C (C3', C5')), 120.94 (4C (C2, C6)), 127.92 (2C (C1')), 129.15 (4C (C2', C6')), 129.36 (4C (C3, C5)), 138.60 (2C (C4)), $149.86(2 \mathrm{C}(\mathrm{C} 1)), 160.50(2 \mathrm{C}(\mathrm{CH}=\mathrm{N})), 162.29$ $\left(2 \mathrm{C}\left(\mathrm{C} 4^{\prime}\right)\right)$.

2.1.4. $N, N^{\prime}$-(methylenebis(4,1-phenylene))bis(1-(4-nitrophenyl)methanimine) (3d): Yellowish color solid, Yield $=81 \%$, M.P $=155-156^{\circ}$ C.FT-IR $\left(\mathrm{cm}^{-1}\right)$ : 3021 (C-H Ar. str. $), 2938,2855$ (C-H Aliph. str.), 1617 ( $\left.\mathrm{C}=\mathrm{N}_{\text {str. imine }}\right), 1593$ ( $\mathrm{C}=\mathrm{C}$ Ar. str.), 1567 $(\mathrm{N}=\mathrm{O}$ asymm. str. $), 1371(\mathrm{~N}=\mathrm{O}$ symm. str. $) .{ }^{1} \mathrm{H}-\mathrm{NMR}$ (DMSO-d $\left.d_{6}\right)$ ppm: $\delta 3.973\left(\mathrm{~s}, 2 \mathrm{H}\left(\mathrm{CH}_{2}\right)\right), 6.889-6.910(\mathrm{~d}, 4 \mathrm{H}(\mathrm{H} 2, \mathrm{H} 6)$, $J=8.4 \mathrm{~Hz}), 6.985-7.005(\mathrm{~d}, 4 \mathrm{H}(\mathrm{H} 3, \mathrm{H} 5), J=8 \mathrm{~Hz}), 7.187-7.207$ (d, 4H (H2', H6'), J=8Hz), 7.791-7.810 (d, 4H (H3', H5'), $J=7.6 \mathrm{~Hz}), 8.508(\mathrm{~s}, 2 \mathrm{H}(\mathrm{CH}=\mathrm{N})) .{ }^{13} \mathrm{C}-\mathrm{NMR}$ (DMSO- $\left.d_{6}\right)$ ppm: $\delta 47.56\left(1 \mathrm{C}\left(\mathrm{CH}_{2}\right)\right), 121.47(4 \mathrm{C}(\mathrm{C} 2, \mathrm{C} 6)), 124.82(4 \mathrm{C}$ (C3', C5')), 127.58 (4C (C2', C6')), 130.08 (4C (C3, C5)),
$139.50(2 \mathrm{C}(\mathrm{C} 4)), 143.88\left(2 \mathrm{C}\left(\mathrm{C}^{\prime}\right)\right), 147.68(2 \mathrm{C}(\mathrm{C} 1), 149.63$ $\left(2 \mathrm{C}\left(\mathrm{C}^{\prime}\right)\right), 161.36(2 \mathrm{C}(\mathrm{C}=\mathrm{N}))$.

2.1.5. $N, N^{\prime}$-(methylenebis(4,1-phenylene))bis(1-(4-chlorophenyl)methanimine) (3e): Deep brown color solid, Yield $=84 \%$, M.P $=142-143^{\circ} \mathrm{C}$. FT-IR $\left(\mathrm{cm}^{-1}\right): 3053$ (C-H Ar. str.), 2926, $2853(\mathrm{C}-\mathrm{H}$ Aliph. str. $), 1630(\mathrm{C}=\mathrm{N}$ str. imine $), 1588(\mathrm{C}=\mathrm{C}$ Ar. str. $) .{ }^{1} \mathrm{H}-\mathrm{NMR}$ (DMSO-d $d_{6}$ ppm: $\delta 4.035\left(\mathrm{~s}, 2 \mathrm{H}\left(\mathrm{CH}_{2}\right)\right), 7.275-7.296(\mathrm{~d}, 4 \mathrm{H}(\mathrm{H} 2$, H6), $J=8.4 \mathrm{~Hz}$ ), 7.312-7.334 (d, $4 \mathrm{H}(\mathrm{H} 3, \mathrm{H} 5), J=8.8 \mathrm{~Hz}), 7.554-$ 7.576 (d, 4H (H3', H5'), $J=8.8 \mathrm{~Hz}), 8.143-8.164$ (d, $J=8.4 \mathrm{~Hz}, 4 \mathrm{H}$ $\left.\left(\mathrm{H} 2^{\prime}, \mathrm{H}^{\prime}\right)\right), 8.889(\mathrm{~s}, 2 \mathrm{H}(\mathrm{CH}=\mathrm{N})) .{ }^{13} \mathrm{C}-\mathrm{NMR}\left(\mathrm{DMSO}-d_{6}\right) \mathrm{ppm}$ : $\delta 46.32\left(1 \mathrm{C}\left(\mathrm{CH}_{2}\right)\right), 121.24\left(4 \mathrm{C}(\mathrm{C} 2, \mathrm{C} 6), 128.29\left(4 \mathrm{C}\left(\mathrm{C}^{\prime}, \mathrm{C}^{\prime}\right)\right.\right.$, $129.60\left(4 \mathrm{C}\left(\mathrm{C}^{\prime}, \mathrm{C}^{\prime}\right)\right), 129.72(4 \mathrm{C}(\mathrm{C} 3, \mathrm{C} 5)), 134.91\left(2 \mathrm{C}\left(\mathrm{C} 1^{\prime}\right)\right)$, $136.62\left(2 \mathrm{C}\left(\mathrm{C}^{\prime}\right)\right), 139.83$ (2C (C4)), 149.12 (2C (C1)), 161.77 $(2 \mathrm{C}(\mathrm{C}=\mathrm{N}))$.

2.1.6. $\quad N, N^{\prime}$-(methylenebis(4,1-phenylene))bis(1-(4-fluorophenyl)methanimine) (3f): Yellowish-orange solid, Yield $=80 \%$, M.P $=250-251^{\circ} \mathrm{C}$. FT-IR $\left(\mathrm{cm}^{-1}\right): 3029$ (C-H Ar. str.), 2918, $2845(\mathrm{C}-\mathrm{H}$ Aliph. str. $), 1614(\mathrm{C}=\mathrm{N}$ str. imine $), 1576(\mathrm{C}=\mathrm{C}$ Ar. str. $) .{ }^{1} \mathrm{H}-\mathrm{NMR}$ (DMSO- $d_{6}$ ) ppm: $\delta 3.885\left(\mathrm{~s}, 2 \mathrm{H}\left(\mathrm{CH}_{2}\right)\right), 6.314-6.335(\mathrm{~d}, 4 \mathrm{H}(\mathrm{H} 2$, H6), $J=8.4 \mathrm{~Hz}$ ), 6.481-6.503 (d, 4H (H3, H5), $J=8.8 \mathrm{~Hz}), 7.187-$ $7.208\left(\mathrm{~d}, 4 \mathrm{H}\left(\mathrm{H}^{\prime}, \mathrm{H}^{\prime}\right), J=8.4 \mathrm{~Hz}\right), 7.389-7.411\left(\mathrm{~d}, 4 \mathrm{H}\left(\mathrm{H} 2^{\prime}\right.\right.$, $\left.\left.\mathrm{H} 6^{\prime}\right), J=8.8 \mathrm{~Hz}\right), 8.620(\mathrm{~s}, 2 \mathrm{H}(\mathrm{CH}=\mathrm{N})) .{ }^{13} \mathrm{C}-\mathrm{NMR}$ (DMSO- $\left.d_{6}\right)$ ppm: $\delta 46.57\left(1 \mathrm{C}\left(\mathrm{CH}_{2}\right)\right), 113.22\left(4 \mathrm{C}\left(\mathrm{C}^{\prime}, \mathrm{C}^{\prime}\right)\right), 120.34(4 \mathrm{C}$ (C2, C6)), 129.76 (4C (C2', C6') ), 129.91 (4C (C3, C5)), 131.15 $\left(2 \mathrm{C}\left(\mathrm{C} 1^{\prime}\right)\right), 139.49(2 \mathrm{C}(\mathrm{C} 4)), 149.58$ (2C (C1)), $160.47(2 \mathrm{C}$ $(\mathrm{C}=\mathrm{N})), 166.32\left(2 \mathrm{C}\left(\mathrm{C}^{\prime}\right)\right)$.

2.1.7. $N, N^{\prime}$-(methylenebis(4,1-phenylene))bis(1-(4-methoxyphenyl)methanimine) (3g): Yellowish color solid, Yield $=88 \%$, M.P $=159-160^{\circ} \mathrm{C}$. FT-IR $\left(\mathrm{cm}^{-1}\right): 3034\left(\mathrm{C}-\mathrm{H}_{\text {Ar. str. }}\right), 2936,2854$ (C$\left.\mathrm{H}_{\text {Aliph. str. }}\right), 1616\left(\mathrm{C}=\mathrm{N}_{\text {str. imine }}\right), 1585(\mathrm{C}=\mathrm{C}$ Ar. str. $), 1123(\mathrm{C}-\mathrm{O}$ str.). ${ }^{1} \mathrm{H}-\mathrm{NMR}$ (DMSO- $\left.d_{6}\right) \mathrm{ppm}: \delta 3.851\left(\mathrm{~s}, 6 \mathrm{H}\left(\mathrm{OCH}_{3}\right)\right), 3.987$ (s, $\left.2 \mathrm{H}\left(\mathrm{CH}_{2}\right)\right), 7.016-7.036\left(\mathrm{~d}, 4 \mathrm{H}\left(\mathrm{H} 3^{\prime}, \mathrm{H} 5^{\prime}\right), J=8 \mathrm{~Hz}\right), 7.123-7.145$ (d, 4H (H2, H6), $J=8.8 \mathrm{~Hz}), 7.251-7.272(\mathrm{~d}, 4 \mathrm{H}(\mathrm{H} 3, \mathrm{H} 5), J=8.4$ $\mathrm{Hz}), 7.861-7.882$ (d, 4H (H2', H6'), $J=8.4 \mathrm{~Hz}), 8.525$ (s, 2H $(\mathrm{CH}=\mathrm{N})) \cdot{ }^{13} \mathrm{C}-\mathrm{NMR}\left(\mathrm{DMSO}-d_{6}\right) \mathrm{ppm}: \delta 45.83\left(1 \mathrm{C}\left(\mathrm{CH}_{2}\right)\right), 55.37$ $\left(2 \mathrm{C}\left(\mathrm{CH}_{3}\right)\right), 114.21\left(4 \mathrm{C}\left(\mathrm{C}^{\prime}, \mathrm{C}^{\prime}\right)\right), 123.06(4 \mathrm{C}(\mathrm{C} 2, \mathrm{C} 6)), 127.39$ (2C (C1')), 128.97 (4C (C2', C6')), 129.40 (4C (C3, C5)), 138.80 (2C (C4)), $149.66(2 \mathrm{C}(\mathrm{C} 1)), 159.30(2 \mathrm{C}(\mathrm{C}=\mathrm{N})), 161.78(2 \mathrm{C}$ $\left.\left(\mathrm{C} 4^{\prime}\right)\right)$.

2.1.8. 4,4 '-((methylenebis(4,1-phenylene))bis(azaneylylidene))bis(methaneylylide-ne) $) \operatorname{bis}(N, N$ - dimethylaniline) (3h): Deep yellowish color solid, Yield $=87 \%$, M.P $=141-142^{\circ}$ C.FT-IR $\left(\mathrm{cm}^{-}\right.$ $\left.{ }^{1}\right): 3039$ (C-H Ar. str.), 2944, 2852 (C-H Aliph. str.), $1612\left(\mathrm{C}=\mathrm{N}_{\text {str. }}\right.$ imine), $1593\left(\mathrm{C}=\mathrm{C}\right.$ Ar. str.). ${ }^{1} \mathrm{H}-\mathrm{NMR}$ (DMSO-d $)$ ppm: $\delta 3.357(\mathrm{~s}$, $\left.12 \mathrm{H}\left(\mathrm{CH}_{3}\right)\right), 3.934\left(\mathrm{~s}, 2 \mathrm{H}\left(\mathrm{CH}_{2}\right)\right), 6.843-6.865\left(\mathrm{~d}, 4 \mathrm{H}\left(\mathrm{H}^{\prime}, \mathrm{H} 5^{\prime}\right)\right.$, $J=8.8 \mathrm{~Hz}), 7.091-7.113(\mathrm{~d}, 4 \mathrm{H}(\mathrm{H} 2, \mathrm{H} 6), J=8.8 \mathrm{~Hz}), 7.173-7.195$ (d, 4H (H3, H5), $J=8.8 \mathrm{~Hz}), 8.069-8.090$ (d, 4H (H2', H6'), $J=$ $8.4 \mathrm{~Hz}), 8.753(\mathrm{~s}, 2 \mathrm{H}(\mathrm{CH}=\mathrm{N}))$. 13C-NMR (DMSO-d6) ppm: $\delta$ $43.79\left(4 \mathrm{C}\left(\mathrm{CH}_{3}\right)\right), 46.23\left(1 \mathrm{C}\left(\mathrm{CH}_{2}\right)\right), 113.98\left(4 \mathrm{C}\left(\mathrm{C}^{\prime}, \mathrm{C}^{\prime}\right)\right)$, $123.92(4 \mathrm{C}(\mathrm{C} 2, \mathrm{C} 6)), 126.34\left(2 \mathrm{C}\left(\mathrm{C}^{\prime}\right)\right), 128.87\left(4 \mathrm{C}\left(\mathrm{C} 2^{\prime}, \mathrm{C}^{\prime}\right)\right)$, 130.08 (4C (C3, C5)), 139.16 (2C (C4)), 149.94 (2C (C1)), $152.26\left(2 \mathrm{C}\left(\mathrm{C} 4^{\prime}\right)\right), 161.17(2 \mathrm{C}(\mathrm{C}=\mathrm{N}))$.

\subsection{Synthetic method for Bis-3-chloro- $\beta$-lactams synthesis} (4a-h)

A suitable synthetic method was used with a slight modification (Khan et al., 2018). To the solution of $N, N^{\prime}$-(methylenebis(4,1phenylene))bis(1-phenylmethanimine) (Schiff base) derivatives $(1 \mathrm{mmol})(\mathbf{3 a}-\mathbf{h})$ in $15 \mathrm{~mL}$ ethanol, $(0.5 \mathrm{mmol})$ of DABCO was added, the mixture was stirred for 30 minutes at $0-5^{\circ} \mathrm{C}$, and at that point drop-wise $(2 \mathrm{mmol})$ chloroacetyl chloride was added, then the mixture was irritated at $25{ }^{\circ} \mathrm{C}$ for about $2-3 \mathrm{~h}$. The reaction's completion was checked through TLC using diethyl ether and ethyl acetate (7:3) as an eluent. Subsequently to the accomplishment of DABCO reaction, $\mathrm{HCl}$ salt was filtered off. The rest was collected and the solvent was vanished through a rotating evaporator. The column chromatography was used to purify the products, by using diethyl ether: $\mathrm{MeOH}$ : ethyl acetate (7:1:2) as eluent. The reaction was illustrated in the scheme (1). 
2.2.1. cis-1,1'-(methylenebis(4,1-phenylene))-cis-bis(3-chloro-4-phenylazetidin-2-one) (4a): Milky color solid, yield= $61 \%$, M.P $=149-150{ }^{\circ} \mathrm{C}$. FT-IR $\left(\mathrm{cm}^{-1}\right): 3039$ (C-H Ar. str.), 2926, 2852 (C-H Aliph. str.), $1670(\mathrm{C}=\mathrm{O}$ str. $\beta$-lactams $), 1584(\mathrm{C}=\mathrm{C}$ Ar. str.). ${ }^{1} \mathrm{H}-\mathrm{NMR}$ (DMSO- $\left.d_{6}\right)$ ppm: $\delta 3.901\left(\mathrm{~s}, 2 \mathrm{H}\left(\mathrm{CH}_{2}\right)\right)$, 5.225-5.236 (d, $2 \mathrm{H}$ (C $\left.\underline{\mathrm{H}}-\mathrm{N}_{\beta \text {-lactams }),}, J=4.4 \mathrm{~Hz}\right), 5.949-5.960$ $\left(\mathrm{d}, 2 \mathrm{H}\left(\mathrm{C} \underline{\mathrm{H}}-\mathrm{Cl}{ }_{\beta \text {-lactams }}\right), J=4.4 \mathrm{~Hz}\right), 6.973-6.995(\mathrm{~d}, 4 \mathrm{H}(\mathrm{H} 2$, H6), $J=8.8 \mathrm{~Hz}), 7.340-7.361$ (d, 4H (H3, H5), $J=8.4 \mathrm{~Hz})$, 7.401 (t, $\left.2 \mathrm{H}\left(\mathrm{H}^{\prime}\right), J=7.2 \mathrm{~Hz}, J=7.2 \mathrm{~Hz}\right), 7.450-7.470$ (d, $4 \mathrm{H}$ $\left.\left(\mathrm{H}^{\prime}{ }^{\prime}, \mathrm{H}^{\prime}\right), J=8 \mathrm{~Hz}\right), 7.512-7.533\left(\mathrm{~d}, 4 \mathrm{H}\left(\mathrm{H}^{\prime}{ }^{\prime}, \mathrm{H} 6^{\prime}\right), J=8.4\right.$ $\mathrm{Hz}) .{ }^{13} \mathrm{C}-\mathrm{NMR}\left(\mathrm{DMSO}-d_{6}\right) \mathrm{ppm}: \delta 45.54\left(1 \mathrm{C}\left(\mathrm{CH}_{2}\right)\right), 61.75$ (2C (C-Cl $\beta$-lactams)), 66.12 (2C (C-N $\beta$-lactam)), 126.99 (2C (C4')), 127.06 (4C (C2', C6')), 128.39 (2C (C4)), 129.89 (4C (C3', C5')), 130.65 (4C (C3, C5), 133.40 (4C (C2, C6), $138.20(2 \mathrm{C}(\mathrm{C} 1)), 145.5\left(2 \mathrm{C}\left(\mathrm{Cl}^{\prime}\right)\right), 162,40\left(2 \mathrm{C}\left(\mathrm{C}=\mathrm{O}_{\beta-}\right.\right.$ lactams)).

2.2.2. cis-1,1'-(methylenebis(4,1-phenylene))-cis-bis(4-(4bromophenyl)-3-chloroazetidin-2-one) (4b): White color solid; yield $=65 \%$, M.P $=169-170{ }^{\circ}$ C. FT-IR $\left(\mathrm{cm}^{-1}\right): 3051(\mathrm{C}-$ $\mathrm{H}$ Ar. str.), 2919, 2847 (C-H Aliph. str.), 1674 ( $\mathrm{C}=\mathrm{O}$ str. $\beta$-lactams), $1581\left(\mathrm{C}=\mathrm{C}_{\text {Ar. str. }}\right){ }^{1} \mathrm{H}-\mathrm{NMR}\left(\mathrm{DMSO}-d_{6}\right) \mathrm{ppm}: \delta 3.837(\mathrm{~s}, 2 \mathrm{H}$ $\left.\left(\mathrm{CH}_{2}\right)\right), 5.265-5.277\left(\mathrm{~d}, 2 \mathrm{H}\left(\mathrm{CH}-\mathrm{N}_{\beta \text {-lactams }}\right), J=4.8 \mathrm{~Hz}\right), 5.842$ $5.854\left(\mathrm{~d}, 2 \mathrm{H}\left(\mathrm{CH}-\mathrm{Cl}_{\beta \text {-lactams }}\right), J=4.8 \mathrm{~Hz}\right), 6.596-6.615(\mathrm{~d}, 4 \mathrm{H}$ $(\mathrm{H} 2, \mathrm{H} 6), J=7.6 \mathrm{~Hz}), 6.771-6.791(\mathrm{~d}, 4 \mathrm{H}(\mathrm{H} 3, \mathrm{H} 5), J=8 \mathrm{~Hz})$, 7.197-7.218 (d, 4H (H2', H6'), J=8.4Hz), 7.471-7.490 (d, 4H (H3', H5'), $J=7.6 \mathrm{~Hz}$ ). ${ }^{13} \mathrm{C}-\mathrm{NMR}$ (DMSO- $d_{6}$ ) ppm: $\delta 42.56$ $\left(1 \mathrm{C}\left(\mathrm{CH}_{2}\right)\right), 61.32\left(2 \mathrm{C}\left(\mathrm{C}^{-C} \mathrm{Cl}_{\beta \text {-lactams }}\right)\right), 69.93\left(2 \mathrm{C}\left(\mathrm{C}-\mathrm{N}{ }_{\beta-}\right.\right.$ lactams)), $123.86\left(2 \mathrm{C}\left(\mathrm{C}^{\prime}\right)\right), 127.51\left(4 \mathrm{C}\left(\mathrm{C} 2^{\prime}, \mathrm{C} 6^{\prime}\right)\right), 128.23$ (2C (C4)), 129.31 (4C (C3, C5)), 130.20 (4C (C3', C5')), 133.67 (4C (C2, C6)), 137.71 (2C (C1)), $143.10\left(2 \mathrm{C}\left(\mathrm{C} 1^{\prime}\right)\right)$, $161.92\left(2 \mathrm{C}\left(\mathrm{C}=\mathrm{O}_{\beta \text {-lactams }}\right)\right)$.

2.2.3. cis-1,1'-(methylenebis(4,1-phenylene))-cis-bis(3-chloro-4-(4-hydroxyphenyl) azetidin-2-one) (4c): Yellow color solid, yield $=68 \%$, M.P $=235-236^{\circ} \mathrm{C}$. FT-IR $\left(\mathrm{cm}^{-1}\right): 3351(\mathrm{OH}$ str.), 3047 (C-H Ar. str.), 2929, 2853 (C-H Aliph. str.), 1681 (C=O str. $\beta$-lactams $), 1586(\mathrm{C}=\mathrm{C}$ Ar. str. $), 1515(\mathrm{C}=\mathrm{C}$ Ar. str. $) .{ }^{1} \mathrm{H}-\mathrm{NMR}$ (DMSO-d $d_{6}$ ) ppm: $\delta 3.717\left(\mathrm{~s}, 2 \mathrm{H}\left(\mathrm{CH}_{2}\right)\right), 4.435-4.447(\mathrm{~d}, 2 \mathrm{H}$ $\left.\left(\mathrm{CH}-\mathrm{N}{ }_{\beta \text {-lactams }}\right), J=4.8 \mathrm{~Hz}\right), 5.643-5.655\left(\mathrm{~d}, 2 \mathrm{H}\left(\mathrm{CH}-\mathrm{Cl}_{\beta-}\right.\right.$ lactams), $J=4.8 \mathrm{~Hz}), 6.830-6.852\left(\mathrm{~d}, 4 \mathrm{H}\left(\mathrm{H}^{\prime}, \mathrm{H} 5^{\prime}\right), J=8.8 \mathrm{~Hz}\right)$, 7.244-7.265 (d, $4 \mathrm{H}(\mathrm{H} 2, \mathrm{H} 6), J=8.4 \mathrm{~Hz}), 7.716-7.735(\mathrm{~d}, 4 \mathrm{H}$ (H3, H5), $J=7.6 \mathrm{~Hz}), 7.762-7.783\left(\mathrm{~d}, 4 \mathrm{H}\left(\mathrm{H} 2^{\prime}, \mathrm{H} 6{ }^{\prime}\right), J=8.4\right.$ $\mathrm{Hz}), 9.175$ (br, s, $2 \mathrm{H}(\mathrm{OH})) \cdot{ }^{13} \mathrm{C}-\mathrm{NMR}$ (DMSO-d $)$ ppm: $\delta$ $41.99\left(1 \mathrm{C}\left(\mathrm{CH}_{2}\right)\right), 56.43\left(2 \mathrm{C}\left(\mathrm{C}-\mathrm{Cl}_{\beta \text {-lactams }}\right)\right), 64.15(2 \mathrm{C}(\mathrm{C}-\mathrm{N}$ $\beta$-lactams $)), 113.63\left(4 \mathrm{C}\left(\mathrm{C}^{\prime}, \mathrm{C} 5^{\prime}\right)\right), 126.42\left(4 \mathrm{C}\left(\mathrm{C} 2^{\prime}, \mathrm{C} 6^{\prime}\right)\right)$, 128.15 (2C (C4)), 130.12 (4C (C3, C5)), 133.28 (4C (C2, C6)), $134.93\left(2 \mathrm{C}\left(\mathrm{C}^{\prime}\right)\right), 137.46(2 \mathrm{C}(\mathrm{C} 1)), 155.66\left(2 \mathrm{C}\left(\mathrm{C} 4^{\prime}\right)\right)$, $161.22\left(2 \mathrm{C}\left(\mathrm{C}=\mathrm{O}_{\beta \text {-lactams }}\right)\right)$.

2.2.4. cis-1,1'-(methylenebis(4,1-phenylene))-cis-bis(3-chloro-4-(4-nitrophenyl) azetidin-2-one) (4d): Milky color solid, yield $=71 \%$, M.P $=182-183{ }^{\circ}$ C. FT-IR $\left(\mathrm{cm}^{-1}\right): 3027(\mathrm{C}-$ $\mathrm{H}$ Ar. str.), 2933, 2849 (C-H Aliph. str.), 1665 (C=O str. $\beta$-lactams), $1585(\mathrm{C}=\mathrm{C}$ Ar. str. $), 1559(\mathrm{~N}=\mathrm{O}$ asymm. str. $), 1366(\mathrm{~N}=\mathrm{O}$ symm. str.). ${ }^{1} \mathrm{H}-\mathrm{NMR}$ (DMSO- $\left.d_{6}\right) \mathrm{ppm}$ : $\delta 3.668\left(\mathrm{~s}, 1 \mathrm{H}\left(\mathrm{CH}_{2}\right)\right), 4.896-$ 4.908 (d, $2 \mathrm{H}\left(\mathrm{CH}^{-\mathrm{N}}{ }_{\beta \text {-lactams }),} J=4.8 \mathrm{~Hz}\right), 5.679-5.691$ (d, $2 \mathrm{H}$ $(\mathrm{CH}-\mathrm{Cl} \beta$-lactams $), \quad J=4.8 \mathrm{~Hz}), \quad 6.793-6.814 \quad(\mathrm{~d}, \quad 4 \mathrm{H} \quad(\mathrm{H} 2$, H6), $J=8.4 \mathrm{~Hz}$ ), 6.972-6.992 (d, 4H (H3, H5), $J=8 \mathrm{~Hz}), 7.335-$ 7.354 (d, 4H (H2', H6'), J=7.6Hz), 7.441-7.462 (d, 4H (H3', $\left.\mathrm{H} 5^{\prime}\right), \quad J=8.4 \mathrm{~Hz}$ ). ${ }^{13} \mathrm{C}-\mathrm{NMR}$ (DMSO- $\left.d_{6}\right)$ ppm: $\delta 43.32(1 \mathrm{C}$ $\left.\left(\mathrm{CH}_{2}\right)\right), 62.81\left(2 \mathrm{C}\left(\mathrm{C}^{-} \mathrm{Cl}_{\beta \text {-lactams }}\right)\right), 69.64\left(2 \mathrm{C}\left(\mathrm{C}-\mathrm{N}_{\beta \text {-lactams }}\right)\right)$, $122.84\left(4 \mathrm{C}\left(\mathrm{C} 2^{\prime}, \mathrm{C}^{\prime}\right)\right), 123.06\left(4 \mathrm{C}\left(\mathrm{C}^{\prime}, \mathrm{C}^{\prime}\right)\right), 127.74(2 \mathrm{C}$ (C4)), 130.29 (4C (C3, C5)), 133.28 (4C (C2, C6)), 137.41 $(2 \mathrm{C}(\mathrm{C} 1)), 144.88\left(2 \mathrm{C}\left(\mathrm{C}^{\prime}\right)\right), 149.19\left(2 \mathrm{C}\left(\mathrm{C} 1^{\prime}\right)\right), 161.93(2 \mathrm{C}$ $\left.\left(\underline{\mathrm{C}}=\mathrm{O}_{\beta \text {-lactams }}\right)\right)$.

2.2.5. trans-1,1'-(methylenebis(4,1-phenylene))-trans-bis(3chl-oro-4-(4-chlorophenyl) azetidin-2-one) (4e): Sandy color, Yield $=73 \%$, M.P $=179-180^{\circ}$ C. FT-IR $\left(\mathrm{cm}^{-1}\right)$ : $3027(\mathrm{C}-$

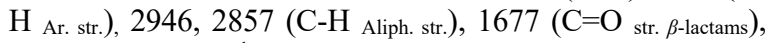
$1597\left(\mathrm{C}=\mathrm{C}\right.$ Ar. str.). ${ }^{1} \mathrm{H}-\mathrm{NMR}\left(\mathrm{DMSO}-d_{6}\right) \mathrm{ppm}: \delta 3.937(\mathrm{~s}, 2 \mathrm{H}$
$\left.\left(\mathrm{CH}_{2}\right)\right), 5.142-5.147$ (d, $2 \mathrm{H}$ (C $\left.\left.\underline{\mathrm{H}-\mathrm{N}}{ }_{\beta \text {-lactams }}\right), J=2 \mathrm{~Hz}\right), 5.821-5.826$ $\left(\mathrm{d}, 2 \mathrm{H}\left(\mathrm{CH}-\mathrm{Cl}_{\beta \text {-lactams }}\right), J=2 \mathrm{~Hz}\right), 6.955-6.975(\mathrm{~d}, 4 \mathrm{H}(\mathrm{H} 2, \mathrm{H} 6)$, $J=8 \mathrm{~Hz}), 7.340-7.361$ (d, 4H (H3, H5), $J=8.4 \mathrm{~Hz}), 7.510-7531$ (d, $\left.4 \mathrm{H}\left(\mathrm{H} 3^{\prime}, \mathrm{H} 5^{\prime}\right), J=8.4 \mathrm{~Hz}\right), 7.583-7.605$ (d, 4H (H2', H6'), $J=8.8$ $\mathrm{Hz}) .{ }^{13} \mathrm{C}-\mathrm{NMR}\left(\mathrm{DMSO}-d_{6}\right) \mathrm{ppm}: \delta 43.60\left(1 \mathrm{C}\left(\mathrm{CH}_{2}\right)\right), 55.45(2 \mathrm{C}$ $\left.\left(\mathrm{C}-\mathrm{Cl}_{\beta \text {-lactams }}\right)\right), 64.34\left(2 \mathrm{C}\left(\mathrm{C}-\mathrm{N}_{\beta \text {-lactams }}\right)\right), 127.84\left(4 \mathrm{C}\left(\mathrm{C} 2^{\prime}, \mathrm{C}^{\prime}\right)\right)$, 128.97 (2C (C4)), 129.45 (4C (C3', C5')), 130.33 (4C (C3, C5)), $132.36\left(2 \mathrm{C}\left(\mathrm{C} 4^{\prime}\right)\right), 134.90(4 \mathrm{C}(\mathrm{C} 2, \mathrm{C} 6)), 137.35$ (2C (C1)), $145.15\left(2 \mathrm{C}\left(\mathrm{C} 1^{\prime}\right)\right), 164.89\left(2 \mathrm{C}\left(\mathrm{C}=\mathrm{O}_{\beta \text {-lactams }}\right)\right)$.

2.2.6. trans-1,1'-(methylenebis(4,1-phenylene))-trans-bis(3-chloro-4-(4-fluorophenyl) azetidin-2-one) (4f): White color solid, yield $=59 \%$, M.P $=155-156^{\circ} \mathrm{C}$. FT-IR $\left(\mathrm{cm}^{-1}\right): 3037$ (C-H Ar. str.), 2932, 2851 (C-H Aliph. str.), $1686\left(\mathrm{C}=\mathrm{O}_{\text {str. } \beta \text {-lactams }}\right), 1593$ (C=C Ar. str.). ${ }^{1} \mathrm{H}-\mathrm{NMR}$ (DMSO- $\left.d_{6}\right) \mathrm{ppm}: \delta 3.956\left(\mathrm{~s}, 2 \mathrm{H}\left(\mathrm{CH}_{2}\right)\right), 5.014-$ $5.019\left(\mathrm{~d}, 2 \mathrm{H}\left(\mathrm{CH}-\mathrm{N}_{\beta \text {-lactams }}\right), J=2 \mathrm{~Hz}\right), 5.596-5.601(\mathrm{~d}, 2 \mathrm{H}(\mathrm{CH}-\mathrm{Cl}$ $\beta$-lactams), $J=2 \mathrm{~Hz}), 6.967-6.987$ (d, $4 \mathrm{H}(\mathrm{H} 2, \mathrm{H6}), J=8 \mathrm{~Hz}), 7.228$ $7.249(\mathrm{~d}, 4 \mathrm{H}(\mathrm{H} 3, \mathrm{H} 5), J=8.4 \mathrm{~Hz}), 7.323-7.342\left(\mathrm{~d}, 4 \mathrm{H}\left(\mathrm{H}^{\prime}, \mathrm{H} 5^{\prime}\right)\right.$, $J=7.6 \mathrm{~Hz}), 7.464-7.486\left(\mathrm{~d}, 4 \mathrm{H}\left(\mathrm{H} 2^{\prime}, \mathrm{H} 5^{\prime}\right), J=8.8 \mathrm{~Hz}\right) .{ }^{13} \mathrm{C}-\mathrm{NMR}$ (DMSO-d $\left.d_{6}\right)$ ppm: $\delta 43.26\left(1 \mathrm{C}\left(\mathrm{CH}_{2}\right)\right), 56.55\left(2 \mathrm{C}\left(\mathrm{C}-\mathrm{Cl}_{\beta \text {-lactams }}\right)\right)$, 66.34 (2C (C-N $\beta$-lactams) $), 117.04$ (4C (C3', C5')), $128.15(2 \mathrm{C}$ (C4)), 130.08 (4C (C2', C6')), 131.93 (4C (C3, C5)), 134.21 (4C (C2, C6), 136.35 (2C (C1)), $139.24\left(2 \mathrm{C}\left(\mathrm{C}^{\prime}\right)\right), 159.36(2 \mathrm{C}$ $\left.\left(\mathrm{C} 4{ }^{\prime \prime}\right)\right), 162.65\left(2 \mathrm{C}\left(\mathrm{C}=\mathrm{O}_{\beta \text {-lactams }}\right)\right)$.

2.2.7. trans-1,1'-(methylenebis(4,1-phenylene))-trans-bis(3-chloro-4-(4-methoxyphenyl) azeti-din-2-one) (4g): Light yellow color solid, yield $=65 \%$, M.P $=176-177^{\circ} \mathrm{C}$. FT-IR: $3037\left(\mathrm{C}-\mathrm{H}_{\mathrm{Ar}}\right.$. str.), 2931, 2848 (C-H Aliph. str.), $1671(\mathrm{C}=\mathrm{O}$ str. $\beta$-lactans $), 1582(\mathrm{C}=\mathrm{C}$ Ar. str.), 1131 (C-O str.). ${ }^{1} \mathrm{H}-\mathrm{NMR}$ (DMSO- $\left.d_{6}\right) \mathrm{ppm}: \delta 3.881(\mathrm{~s}, 6 \mathrm{H}$ $\left.\left(\mathrm{OCH}_{3}\right)\right), 4.266\left(\mathrm{~s}, 1 \mathrm{H}\left(\mathrm{CH}_{2}\right)\right), 5.037-5.041\left(\mathrm{~d}, 2 \mathrm{H}\left(\mathrm{CH}_{-} \mathrm{N}_{\beta \text {-lactams }}\right)\right.$, $J=1.6 \mathrm{~Hz}), 5.528-5.532\left(\mathrm{~d}, 2 \mathrm{H}\left(\mathrm{CH}-\mathrm{Cl}_{\beta \text {-lactams }}\right), \mathrm{J}=1.6 \mathrm{~Hz}\right), 6.954-$ 6.975 (d, 4H (H5', H3'), J=8.4Hz), 7.140-7.160 (d, 4H (H2, H6), $J=8 \mathrm{~Hz}), 7.380-7.402(\mathrm{~d}, 4 \mathrm{H}(\mathrm{H} 3, \mathrm{H} 5), J=8.8 \mathrm{~Hz}), 7.495-7.515$ (d,4H (H2', H6'), $J=8 \mathrm{~Hz}$ ). ${ }^{13} \mathrm{C}-\mathrm{NMR}$ (DMSO- $d_{6}$ ) ppm: $\delta 41.97$ $\left(1 \mathrm{C}\left(\mathrm{CH}_{2}\right)\right), 52.16\left(2 \mathrm{C}\left(\mathrm{CH}_{3}\right)\right), 58.26\left(2 \mathrm{C}\left(\mathrm{C}-\mathrm{Cl}_{\beta \text {-lactams }}\right)\right), 67.74$ (2C (C-N $\beta$-lactams) $), 115.55\left(4 \mathrm{C}\left(\mathrm{C}^{\prime}, \mathrm{C}^{\prime}\right)\right), 126.78$ (4C (C2', C6')), 127.70 (2C (C4)), 129.84 (4C (C3, C5)), 133.30 (4C (C2, C6) $), 136.46\left(2 \mathrm{C}\left(\mathrm{C1}^{\prime}\right)\right), 137.34(2 \mathrm{C}(\mathrm{C} 1)), 159.74\left(2 \mathrm{C}\left(\mathrm{C}^{\prime}\right)\right)$, $163.85\left(2 \mathrm{C}\left(\mathrm{C}=\mathrm{O}_{\beta \text {-lactams }}\right)\right)$.

2.2.8. trans-1,1'-(methylenebis(4,1-phenylene)- trans-bis(3-chloro-4-(4-(dimethylamino) phenyl) azetidin-2-one) (4h): Red color solid, yield $=60 \%$, M.P $=210-211^{\circ} \mathrm{C}$. FT-IR $\left(\mathrm{cm}^{-1}\right): 3035(\mathrm{C}-$

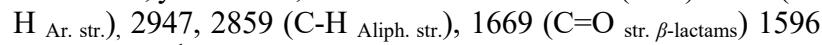
$\left(\mathrm{C}=\mathrm{C}\right.$ Ar. str.). ${ }^{1} \mathrm{H}-\mathrm{NMR}$ (DMSO-d $)$ ppm: $\delta 3.714\left(\mathrm{~s}, 12 \mathrm{H}\left(\mathrm{CH}_{3}\right)\right)$, 4.299 (s, 2H $\left(\mathrm{CH}_{2}\right)$ ), 4.956-4.962 (d, 2H (CH-N $\beta$-lactams), $J=2.4 \mathrm{~Hz}), 5.539-5.533$ (d, $\left.2 \mathrm{H}\left(\mathrm{CH}-\mathrm{Cl}_{\beta \text {-lactams }}\right), J=2.4 \mathrm{~Hz}\right), 6.969$ 6.990 (d, 4H (H3', H5'), J=8.4Hz), 7.466-7.485 (d, 4H (H2, H6), $J=7.6 \mathrm{~Hz}), 7.712-7.732$ (d, $4 \mathrm{H}(\mathrm{H} 3, \mathrm{H} 5), J=8 \mathrm{~Hz}), 7.852-7.871$ (d, $\left.4 \mathrm{H}\left(\mathrm{H}^{\prime}, \mathrm{H}^{\prime}\right), J=7.6 \mathrm{~Hz}\right) .{ }^{13} \mathrm{C}-\mathrm{NMR}$ (DMSO-d $)$ ppm: $\delta 41.97$ $\left(4 \mathrm{C}\left(\mathrm{CH}_{3}\right)\right), 44.02\left(1 \mathrm{C}\left(\mathrm{CH}_{2}\right)\right), 60.52\left(2 \mathrm{C}\left(\mathrm{C}-\mathrm{Cl}_{\beta \text {-lactams }}\right)\right), 70.43$ (2C (C-N $\beta$-lactams)), 113.34 (4C (C3', C5')), 128.94 (2C (C4)), $129.63\left(4 \mathrm{C}\left(\mathrm{C} 2^{\prime}, \mathrm{C}^{\prime}\right)\right), 130.88(4 \mathrm{C}(\mathrm{C} 3, \mathrm{C} 5)), 132.73\left(2 \mathrm{C}\left(\mathrm{C} 1^{\prime}\right)\right)$, 135.33 (4C (C2, C6)), 138.08 (2C (C1)), 149.72 (2C (C4')), $163.39\left(2 \mathrm{C}\left(\mathrm{C}=\mathrm{O}_{\beta \text {-lactams }}\right)\right)$.

\subsection{In Vitro antimicrobial (antibacterial and antifungal) assay:}

The stock microdilution technique was used to test the antibacterial and antifungal measures. The compounds (3a-h) and (4a-h) were screened with an incredible ability to restrain strains in opposition to strains that are gram-positive (S. aureus), and strains that are gram-negative (E. coli) of bacterial and A. Niger (natural isolates). A typical prescription, Amoxicillin and, Penicillin were utilized as standard medications (Aziz and Azeez, 2020).

\subsection{Molecular docking software}

PubChem (https://pubchem.ncbi.nlm.nih.gov/) and ChemDraw Professional 16.0 were utilized to plan the synthesized mixtures (ligands) as the SDF type file format, Data Bank of Protein 
(http://www.rcsb.org/), instruments that are online were utilized for download of a protein (PDB ID code: 3GI9). The zinc (http://zinc.docking.org/) site was utilized for the plan and download of standard medications (amoxicillin and penicillin G). PyRx (AutoDockTools-1.5.6) and BIOVIA Discovery Studio 2020 were operated to envision and adjust receptor and ligand structures (Beg and Athar, 2020, My et al., 2011).

\section{RESULTS AND DISCUSSION}

\subsection{Chemistry}

The preliminary material of 4,4'-methylenedianiline (1) was used for the synthesis of $N, N^{\prime}$-(methylenebis $(4,1-$ phenylene)) bis(1-phenylmethanimine) derivatives (3a-h) via the reaction with different substituted benzaldehydes (2ah), the absolute ethanol was used as a solvent and with few drops of acetic acid as a catalyst. Subsequently, new series bis-3-chloro- $\beta$-lactam derivatives were synthesized by $2+2$ cycloaddition reaction of symmetrical bis-Schiff bases with ketene, the ketene was created of the chloroacetyl chloride's reaction with DABCO (Khan et al., 2018).

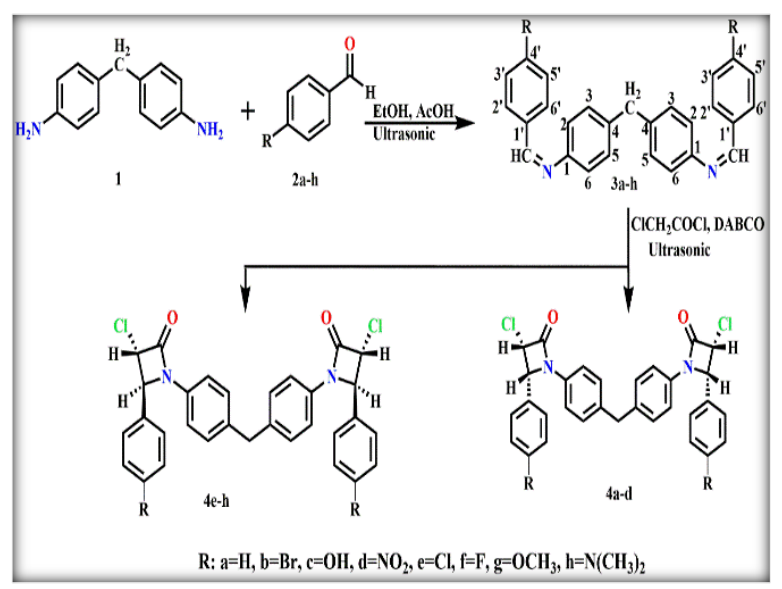

Scheme 1. Synthesis of bis-3-chloro- $\beta$-lactam derivatives

(4a-h).

The produced compound's skeleton was affirmed by FT-IR, ${ }^{1} \mathrm{H}$, and ${ }^{13} \mathrm{C}-\mathrm{NMR}$ spectroscopies. The FT-IR of the bisSchiff bases (3a-h) presented different bands, the important band's region is $1630-1612 \mathrm{~cm}^{-1}$ that refers to the $\mathrm{C}=\mathrm{N}$ of imines extending vibration, and the disappearance of $\left(\mathrm{NH}_{2}\right)$ and $(\mathrm{C}=\mathrm{O})$ bands in 4,4'-methylenedianiline and various substituted benzaldehydes, respectively, confirm the formation of bis-Schiff bases (3a-h). The ${ }^{1} \mathrm{H}-\mathrm{NMR}$ spectra for the bis-imine derivatives (3a-h) showed a signal for the proton of $(\mathrm{C} \underline{\mathrm{H}}=\mathrm{N})$ groups at $(8.889-8.443) \mathrm{ppm}$, while the aromatic protons have appeared at the region (7.81-7.23) ppm. In ${ }^{13} \mathrm{C}$-NMR spectra of the bis-Schiff base derivatives (3a-h), showed a signal for the carbon of $(\mathrm{C}=\mathrm{N})$ groups at (161.77-159.30) ppm, which also confirm the formation of the bis-Schiff base.

The general feature of the FT-IR absorption bands of bis-3chloro- $\beta$-lactam derivatives $(\mathbf{4 a - h})$, showed the disappearance of absorption bands of $(-\mathrm{C}=\mathrm{N})$ and appearance of bands at $1686-1665 \mathrm{~cm}^{-1}$ which belong to carbonyl groups of bis-3-chloro- $\beta$-lactam, this evidence confirmed the formation of the desired product. The ${ }^{1} \mathrm{H}-\mathrm{NMR}$ chemical shifts for bis-3-chloro- $\beta$-lactam derivatives (4a-h) showed a double in the region (5.27-4.45) ppm for $\left(\mathrm{C}^{\mathrm{H}}-\mathrm{N} \beta\right.$-lactams $)$ groups of bis-3-chloro- $\beta$-lactam, which a singlet in the region (8.89-8.44) ppm in $(\mathrm{C} \underline{H}=\mathrm{N})$ groups of bis-imines (3a-h) were disappeared, and a doublet has appeared in the region (5.955.53) ppm for ( $\left.\underline{\mathrm{CH}}-\mathrm{Cl}_{\beta \text {-lactams }}\right)$, all these evidences confirm the obtaining of the desired bis-3-cholro- $\beta$-lactam compounds. The ${ }^{13} \mathrm{C}-\mathrm{NMR}$ spectra for the synthesized compounds (4a-h) showed that the carbon of carbonyl groups of bis-3-chloro- $\beta$-lactam appeared at $(164.89-161.22) \mathrm{ppm}$, in addition to a signal for $\left(\mathrm{C}-\mathrm{N}_{\beta \text {-lactams }}\right)$ and $\left(\mathrm{C}-\mathrm{Cl}_{\beta \text {-lactams }}\right)$ carbon groups that appeared at (70.43-64.15) ppm and (62.81-55.45) ppm, respectively.

Two new $\mathrm{C} 3$ and $\mathrm{C} 4$ stereocenters in the $\beta$-lactams ring are created via the reaction of a mono-substituted ketene and an acyclic imine, thus the creation could be cis, trans configuration (Jocabed Marquez 2012). The relative cis and trans stereoselectivity is measured as a serious matter in the reaction of Staudinger. The stereochemical assignment based on characteristic ${ }^{1} \mathrm{H}-\mathrm{NMR}$ patterns displayed by the two isomeric series, for the synthesized bis-3-cholro- $\beta$-lactams (4a-d) the $J$ value for $\underline{\mathrm{C}} \underline{\mathrm{H}}-\mathrm{N}$ lactams and $\mathrm{C} \underline{\mathrm{H}}-\mathrm{Cl}$ lactams are equal to $4.4 \mathrm{~Hz}$ and $4.8 \mathrm{~Hz}$ that means cis configuration while in the compounds $(4 \mathrm{e}-$ h) the $J$ value for $\mathrm{C} \underline{\mathrm{H}}-\mathrm{N}$ lactams and $\mathrm{C} \underline{\mathrm{H}}-\mathrm{Cl}_{\text {lactams }}$ is equal to $1.6 \mathrm{~Hz}$, $2 \mathrm{~Hz}$ and $2.4 \mathrm{~Hz}$ that means trans configuration, though the value seems to be always larger for the cis-isomer contrast to its transcomplement(McLoughlin et al., 2019, Méndez et al., 2018).

\subsection{Biological activity}

\subsubsection{In vitro Antimicrobial assay}

The in vitro antimicrobial bis-Schiff bases activities (3a-h) and bis-3-chloro- $\beta$-lactams (4a-h) were observed against grampositive strain (Staphylococcus aureus (ATCC 9144)), and gramnegative strains (Escherichia coli (ATCC 8739)). In addition, antifungal activity in opposition to Aspergillus Niger and Trichophyton mentagrophytes was evaluated. The antimicrobial of the synthesized compounds assessed through the broth microdilution technique; the results were detailed by the (MIC) minimum inhibitory concentration as shown in (Table 1).

The synthesized bis-Schiff bases (3a-h) were screened with higher antibacterial and antifungal activities as compared to standards penicillin $\mathrm{G}$, and nystatin drugs, respectively. Compound (3a, 3b) exhibited the most elevated antibacterial activity in opposition to Escherichia coli and Staphylococcus aureus bacteria and showed the most remarkable activity against Aspergillus Niger and compound $(\mathbf{3 b}, \mathbf{3 f})$ against Trichophyton mentagrophytes fungi, while compound (3c, 3d) exhibited the lowest antibacterial activity in opposition to Escherichia coli and Staphylococcus aureus bacteria. Compounds (3c, 3d) and (3a, 3c) displayed the lowest antifungal activity in opposition to Aspergillus Niger and Trichophyton mentagrophytes fungi.

The bis-3-chloro- $\beta$-lactams' (4a-h) antifungal and antibacterial activities compared to standards (penicillin G, and nystatin) drugs showed higher activities. (4a-h) compared to standards (penicillin G, and nystatin) drugs showed higher activities. Compound $(\mathbf{4 a}, \mathbf{4 b})$ exhibited the most elevated antibacterial activity in opposition to Escherichia coli and Staphylococcus aureus bacteria and showed the most remarkable activity against Aspergillus Niger and Trichophyton mentagrophytes fungi, while compound $(\mathbf{4 c}, \mathbf{4 d})$ exhibited the lowest antibacterial activity against Escherichia coli, Staphylococcus aureus bacteria, also against Aspergillus Niger and Trichophyton mentagrophytes fungi, as shown in Table 1. 
Table 1. In vitro antimicrobial assay of the synthesized compounds

\begin{tabular}{|c|c|c|c|c|}
\hline \multirow{2}{*}{ Entry } & \multicolumn{3}{|c|}{ MIC for Microorganisms $(\boldsymbol{\mu g} / \mathbf{m l})$} \\
\cline { 2 - 5 } & Bacterial strains & \multicolumn{2}{|c|}{ Fungal strains } \\
\cline { 2 - 5 } 3a & S. & $\boldsymbol{E}$. & $\boldsymbol{A}$. & $\boldsymbol{T}$. \\
Aureus & Coli & Niger & Mentagrophytes \\
$\mathbf{3 b}$ & 3.70 & 3.45 & 5.90 & 4.60 \\
$\mathbf{3 c}$ & 3.60 & 3.40 & 5.85 & 5.45 \\
$\mathbf{3 d}$ & 2.70 & 2.50 & 5.0 & 4.75 \\
$\mathbf{3 e}$ & 2.80 & 2.60 & 5.20 & 4.90 \\
$\mathbf{3 f}$ & 3.35 & 3.0 & 5.75 & 5.25 \\
$\mathbf{3 g}$ & 3.50 & 3.25 & 5.80 & 5.30 \\
$\mathbf{3 h}$ & 3.0 & 2.75 & 5.5 & 5.0 \\
$\mathbf{4 a}$ & 3.25 & 2.90 & 5.60 & 5.20 \\
$\mathbf{4 b}$ & 2.5 & 2.30 & 4.75 & 4.5 \\
$\mathbf{4 c}$ & 2.25 & 2.0 & 4.60 & 4.20 \\
$\mathbf{4 d}$ & 0.90 & 0.70 & 3.5 & 3.10 \\
$\mathbf{4 e}$ & 0.95 & 0.80 & 3.7 & 3.30 \\
$\mathbf{4 f}$ & 1.80 & 1.60 & 4.40 & 3.75 \\
$\mathbf{4 g}$ & 2.0 & 1.70 & 4.45 & 3.85 \\
$\mathbf{4 h}$ & 1.50 & 1.20 & 3.90 & 3.50 \\
Penicillin G & 1.75 & 1.50 & 4.20 & 3.70 \\
Nystatin & 0.25 & 0.10 & - & - \\
\hline
\end{tabular}

\subsubsection{Molecular Docking study}

A study of the molecular docking was used to recognize the binding model of the newly synthesized mixtures and the molecule target (Patel HM, 2019). For this reason, the target proteins or receptors of $E$. coli (PDB ID: 3GI9) were selected to be a likeable target of molecular docking (Mohatnta et al., 2014). The synthesized bis-Schiff bases (3a-h) showed in Table(2), and the newly synthesized bis-3-chloro- $\beta$-lactams (4a-h) showed in Table (3) were docked into the binding active site of the protein, and the anticipated energies of the binding which have little lower than the standard in case of bis-Schiff bases but lower in case of bis-3-chloro- $\beta$-lactams, and in the majority of the cases, the connections of the hydrogen bonding were noted within the mixtures and penicillin-binding protein 3GI9, besides pi-cation, pi-alkyl, pi-sulfur, pi-sigma, and pi-pi T-shaped, Pi-Pi Stacked was also observed with the target protein.

Table 2. Docking results of synthesized Schiff bases (3a-h) docked with E. coli (PDB ID: 3GI9)

\begin{tabular}{|c|c|c|}
\hline Entry & $\begin{array}{c}\Delta G \text { bind } \\
\text { (kcal/mol) }\end{array}$ & Interactions \\
\hline $3 \mathbf{a}$ & -8.1 & $\begin{array}{l}\text { Van der Waals: ASP H:52, ASP H:55, SER C:320, LYS C:319, PHE C:318, HIS H:101, TYR H:103, THR H:105, } \\
\text { TYR H:107, ASN H:108. Conventional hydrogen bond: TYR H:102. Pi-Donor hydrogen bond: THR C:321, GLY } \\
\text { H:104, THR H:105, TYR H:107, ASN H:108. Pi-Pi stacked: TYR H:57 }\end{array}$ \\
\hline $\mathbf{3 b}$ & -7.7 & $\begin{array}{l}\text { Van der Waals: SER C:320, TYR H:103, ASP H:52, HIS H:101, PHE C:318, THR C:321, GLY H:104, TRP H:33, } \\
\text { THR H:105, TYR H:107, ASN H:108, THR H:106. Conventional hydrogen bond: ASP H:55. Pi-Sigma: LYS C:319. } \\
\text { Pi-Pi Stacked: TYR H:57 }\end{array}$ \\
\hline $3 c$ & -7.3 & $\begin{array}{l}\text { Van der Waals: SER H:54, TYR H:103, THR H:105, HIS H:101, PHE C:318, GLY H:104, TRP H:33, TYR L:102, } \\
\text { GLY H:104, SER C:320, THR H:105, SER H:54, TYR L: 102.Conventional hydrogen bond: THR C:321, ASP H:55, } \\
\text { ASP H:52, ASN H:108. Pi-Donor hydrogen: GLU H:50, TYR H: 57. Pi-Sigma: HIS H: 101. Pi-Pi Stacked: TYR } \\
\text { H:57. Pi-Pi T-Shaped: GLU H:50. Pi-Anion: GLU H: 50. Carbon Hydrogen bond: GLU H:50. }\end{array}$ \\
\hline 3d & -8.5 & $\begin{array}{l}\text { Van der Waals: GLU C:2, LEU C:3, TYR H:102, GLU C:313, THR H:105, THR H:106, ASN L:34, LYS L:36, THR } \\
\text { L:59, TYR H:109. Conventional Hydrogen Bond: TYR L:55, LYS C:145, LYS C:4. Pi-Cation: LYS C:6. Pi-Donor } \\
\text { Hydrogen Bond: TYR H:107, TYR H:107. Pi-Pi Stacked: PHF L:56. Pi-Pi T-Shaped: PHF L:56. Pi-Alkyl: LYS } \\
\text { C:4. }\end{array}$ \\
\hline $3 e$ & -7.3 & $\begin{array}{l}\text { Van der Waals: LEU C:360, PHF C:380, LEU C:384, PHF C:387. Pi-Sigma: LEU C:356. Pi-Pi-T-Shaped: PHE C: } \\
\text { 409.Alkyl and Pi-alkyl: VAL C:353, PHE C:357, LEU C:89, VAL C:353, LEU C:413, ALA C:88. }\end{array}$ \\
\hline $3 f$ & -9.2 & $\begin{array}{l}\text { Van der Waals: LEU C:333, PHE C:334, VAL C:412, TRP C:94, TYR C:300, TYR C:419, ILE C:91. Conventional } \\
\text { Hydrogen Bond: GLY C:411. Halogen (Fluorine): ALA C:303. Pi-Sigma: ILE C: } 326 \text {. Pi-Sulfur: MET C:95. Pi- } \\
\text { Pi Stacked: PHE C: } 415 \text {. Pi-Pi T-Shaped: PHE C:415. Pi-Alkyl: LEU C:330, LYS C:304 }\end{array}$ \\
\hline $3 g$ & -8.4 & $\begin{array}{l}\text { Van der Waals: TYR L:102, ASN H:59, THR L:100, ASN H:108, THR H:105, HIS H:101, TYR H:103, PHE C:318, } \\
\text { THR C:321, TRP H:33. Conventional hydrogen bond: ASP H:55, TYR H:102. Pi-Donor: TYR H:107, GLY H:104, } \\
\text { PHE C:318, SER C:320, GLU H:50. Pi-Pi Stacked: TYR H:57. Pi-Alkyl: LYS C:319. }\end{array}$ \\
\hline $3 h$ & -7.9 & $\begin{array}{l}\text { Van der Waals: TRP H:33, TYR H:103, LYS C:319, SER C:320, PHE C:318, GLY H:104, TRP C:317. Conventional } \\
\text { Hydrogen bond: ASP H:52, THR C:321. Pi-Donor Hydrogen Bond: ASP H:55, ASN C:140, PHE C:141 }\end{array}$ \\
\hline
\end{tabular}

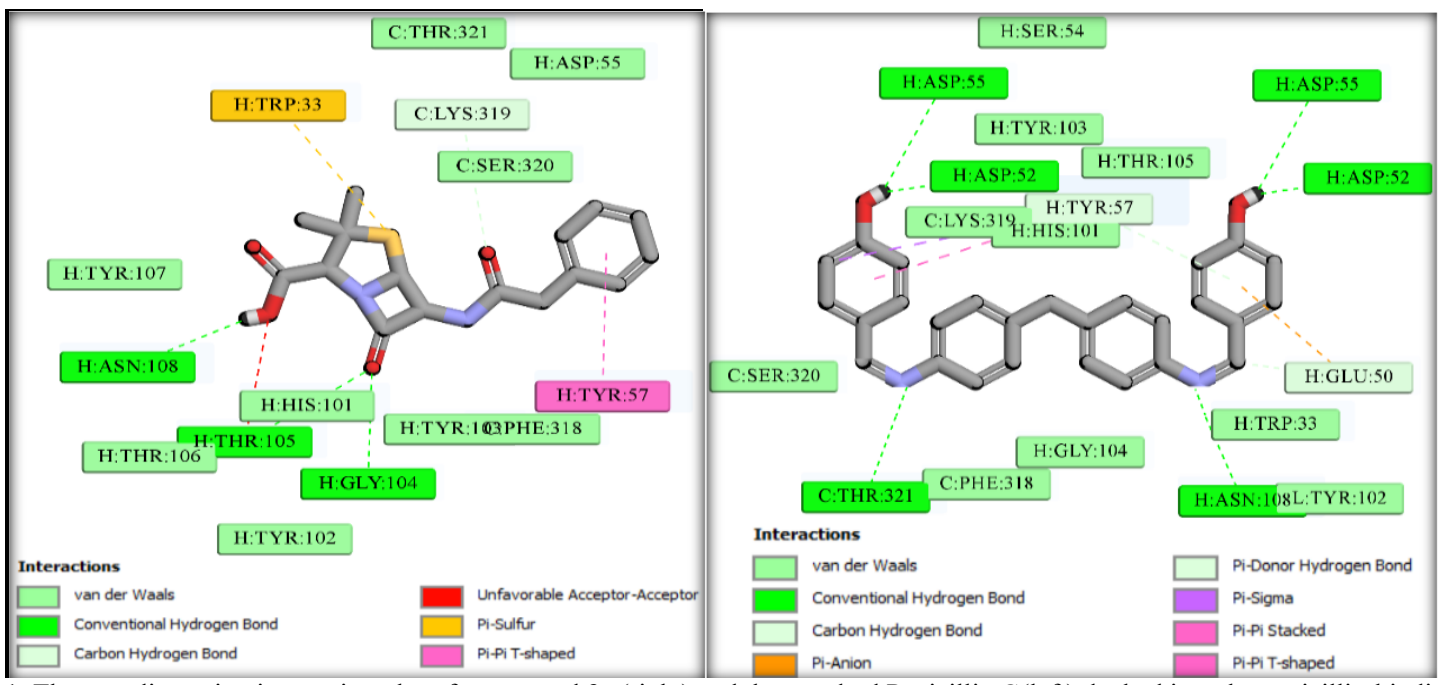

Figure 1. The two-dimension interaction plot of compound 3c (right) and the standard Penicillin G(left) docked into the penicillin-binding protein's active site (PDB ID: 3GI9). 

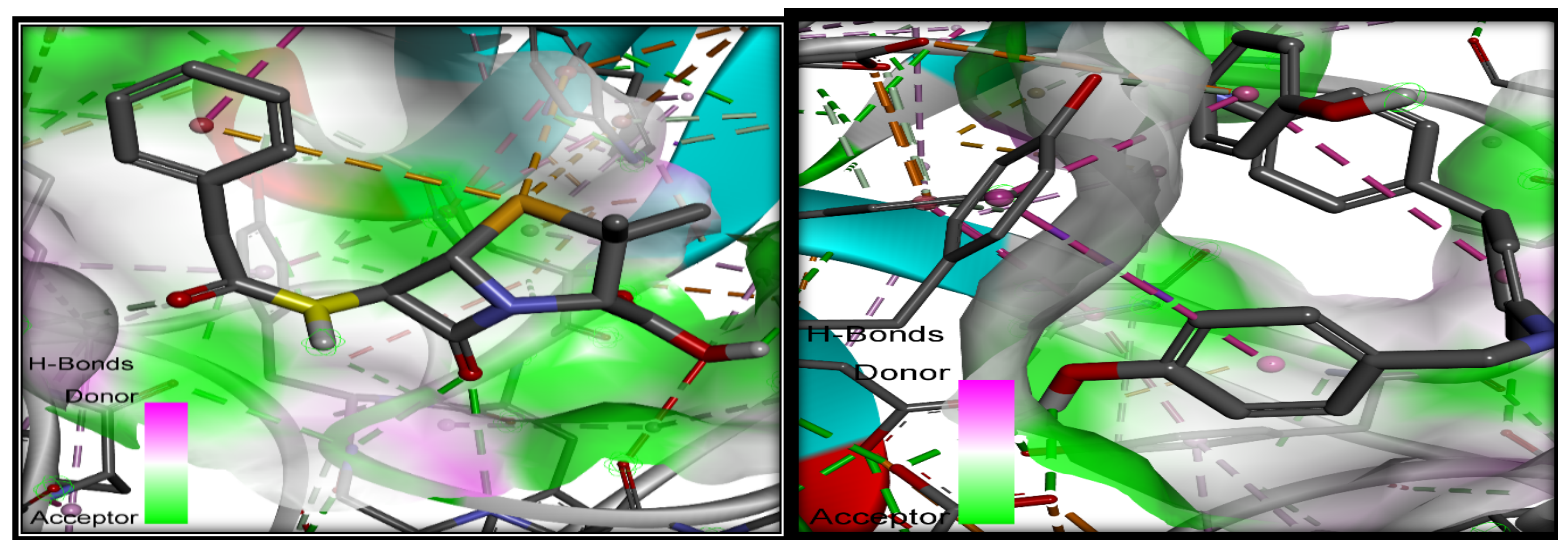

Figure 2. The three-dimension interaction plot of compound 3c (right) and the standard Penicillin G (left) docked into the penicillin-binding protein's active site (PDB ID: 3GI9)

As revealed in Table (2) compound $(\mathbf{3 c}, \mathbf{3 e})$ was the most potent derivatives and their binding energy was close to the standard, while compound (3f, 3d) was the least potent one. These outcomes are compatible with the noted antibacterial outcomes. On the subject of bis-2-lactams shown in Table (3), compound $4 \mathbf{e}$ was the potent derivatives and compound $4 \mathbf{a}$ was the least potent one. The outcomes evidenced that the synthesized bis-Schiff base (3a-h) were the most potent compounds compared to the synthesized bis-2-lactams (4a-h).

\begin{tabular}{|c|c|c|}
\hline Entry & $\begin{array}{l}\Delta \mathrm{G} \mathrm{bind} \\
\text { (kcal/m } \\
\text { ol) }\end{array}$ & Interactions \\
\hline $4 a$ & -9.8 & $\begin{array}{l}\text { Van der Waals: TYR C:419, GLU C:322, MET C:95, THR C:408, PHE C:334, VAL C:412, GLY C:411, TRP } \\
\text { C:94. Conventional hydrogen bond: TYR C: 300.Pi-Sigma: ILE C:326. Pi-Pi stacked: PHE C:415. Pi-Alkyl: } \\
\text { ALA C:303, ILE C:91, LYS C:304, LEU C:330. }\end{array}$ \\
\hline $4 b$ & -8.5 & $\begin{array}{l}\text { Van der Waals: GLU C:313, LYS C:145, LYS C:315, GLY C:148, GLU C:151, PHE C:152, GLU C:12, LYS C:4, } \\
\text { LYS C:7, LEU C:8. Pi-Sigma: LYS C:6. Alkyl: TYR H:107, TYR H:102. Pi-Alkyl: ARG C:149. }\end{array}$ \\
\hline $4 c$ & -8.6 & $\begin{array}{l}\text { Van der Waals: TRP H:33, TYR H:57, SER C:320, LYS C:319, PHE C:318, THF C:321, TRP C:317, ASN C:140, } \\
\text { TYR H:103, HIS H:101, PHE C:141, PHE C:142, LYS C:145. Conventional Hydrogen Bond: THR H:105, GLY } \\
\text { H:104, TYR H:102. Pi-Sigma: THR H:106. Pi-Alkyl: VAL C:316. }\end{array}$ \\
\hline $4 d$ & -8.2 & $\begin{array}{l}\text { Van der Waals: ASN H:108, TRP H:33, TYR H:103, ASP H:52, THR H:105, ASN C:140, TRP C:317, THR } \\
\text { C:321, LYS C:304, PHE C:318, GLU C:322, SER C:320, GLY H:104, LYS C:319.Pi-Anion: ASP H:55, Pi-Pi T- } \\
\text { shaped: HIS H:101, TYR H:57. }\end{array}$ \\
\hline $4 e$ & -8.1 & $\begin{array}{l}\text { Van der Waals: LEU C:384, PHF C:387, PHE C:409, LEU C:92, LEU C:92, LEU C:413. Pi-Pi-Pi T-Shaped: } \\
\text { PHE C:380. Alkyl: PHE C:357, ILE C:85, ALA C:88, LEU C:89. Pi-alkyl: LEU C:360, LEU C:356, VAL C:353. }\end{array}$ \\
\hline $4 f$ & -8.5 & $\begin{array}{l}\text { Van der Waals: LEU C:360, PHE C:357, PHE C:387, VAL C:412, LEU C:92, LEU C:413. Pi-Sigma: VAL C:353. } \\
\text { Pi-Pi T-Shaped: PHE C:409. PHE C:380. Pi-Alkyl: LEU C:356, ILE C:85. }\end{array}$ \\
\hline $4 \mathrm{~g}$ & -8.6 & 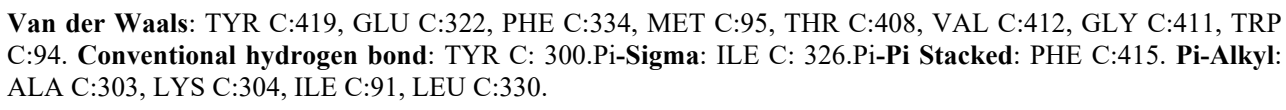 \\
\hline $4 h$ & -8.4 & $\begin{array}{l}\text { Van der Waals: VAL C:316, TRP C:317, THR H:105, ASN C:140, PHE C:141, PHE C:318, TYR H:103, SER } \\
\text { C:320, LYS C:319, THR C:321, ASP H:55. Carbon hydrogen bond: HIS H:101, Pi-Pi Stacked and Amide-Pi- } \\
\text { Stacked: GLY H:104, TYR H:57. Pi-Alkyl: TRP H:33 }\end{array}$ \\
\hline $\begin{array}{l}\text { Penicillin } \\
\text { G }\end{array}$ & -7.2 & $\begin{array}{l}\text { Van der Waals: TYR H:107, THR C:321, ASP H:55, SER C:320, PHE C:318, THR H:106. Conventional } \\
\text { hydrogen bond: ASN H:108, THR H:105, GLY H:104. Carbon hydrogen bond: LYS C:319, TYR H:102, TYR } \\
\text { H:103. Unfavourable Acceptor- Acceptor: THR H:105. Pi-Sulfur: TRP H:33. Pi-Pi T-Shaped: TYR H:57 }\end{array}$ \\
\hline
\end{tabular}

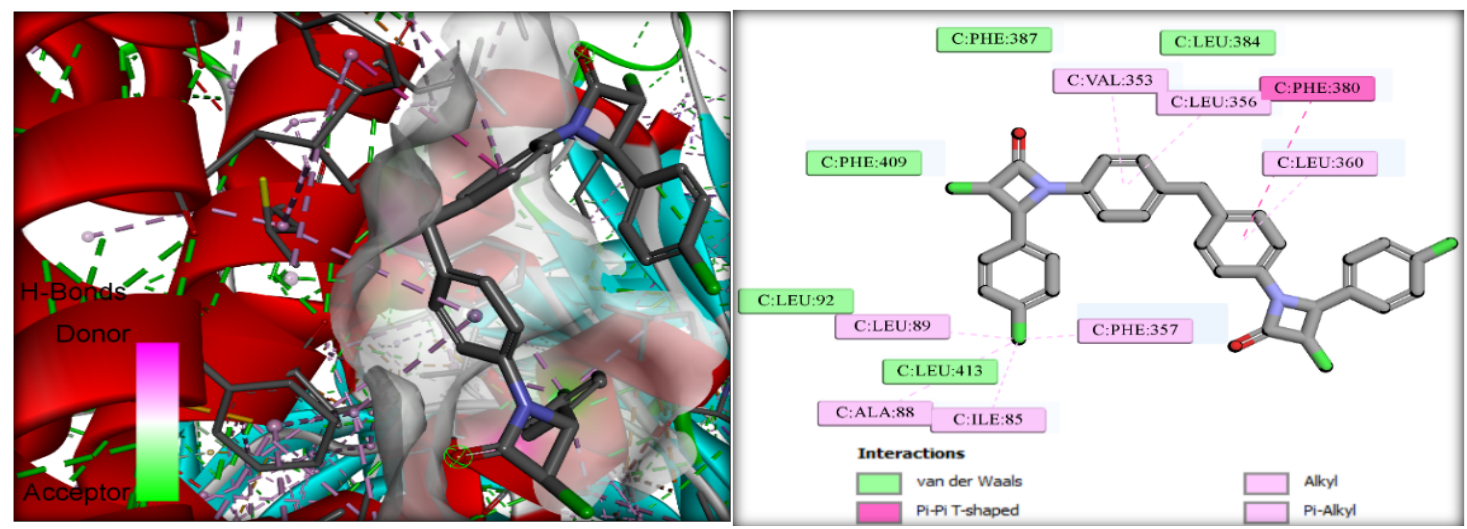

Figure 3.The two-dimension (right) and three-dimensions (left) interaction plot of compound (4e) docked into the penicillin-binding protein's active site (PDB ID: 3GI9). 
The strained four-membered heterocyclic $\beta$-lactam's biological activity is severely reliant on substituent groups' nature which distresses the reaction to the active sites of the molecular, growing or dropping the likelihood of interacting with the substrates (Troisi et al., 2010).

\section{CONCLUSION}

In conclusion, an appropriate synthetic pathway is applied for the synthesis of bis-3-chloro- $\beta$-lactams which gave a good yield. The antimicrobial activity of the synthesized compounds (3a-h) and (4a-h) were respectable in comparison with the standard reference drug. According to the antimicrobial outcomes the synthesized bis-Schiff bases (3a-h) and bis-3chloro- $\beta$-lactams (4a-h) exhibited highest antimicrobial activity which was reinforced by molecular docking study. The synthesized compounds showed good binding energies and interactions. Compound $(\mathbf{3 c}, \mathbf{3 e})$ screened better binding energy and most active to create interaction with receptor sites. The predicted binding energies of bis-Schiff bases have little lower than the standard drugs, while lower in case of bis-3-chloro- $\beta$ lactams, which talent the interaction with the protein receptor. The reactivity to the active sites of the molecular is affected by the substituent groups' nature, and for the majority of cases, interactions due to hydrogen bonding were observed among the synthesized mixtures and penicillin-binding protein 3GI9 besides other interactions. Finally, the newly synthesized compounds might have more biological activities and might be used in pharmaceutical fields after further assessments.

\section{ACKNOWLEDGEMENTS}

The authors would like to express their sincere thanks to the Chemistry department/college of Science Raparin University, and Salahaddin University-Erbil.

\section{REFERENCES}

ABOU-MELHA, K. \& FARUK, H. 2008. Bimetallic complexes of schiff base bis-[4-hydroxycuomarin-3-yl]-1 N, $5 \mathrm{~N}$ thiocarbohydrazone as a potentially dibasic pentadentate ligand. Synthesis, spectral, and antimicrobial properties. Journal of the Iranian Chemical Society, 5, 122-134.

AL-AZZAWI, A. \& AL-OBIADI, K. 2016. Synthesis and antimicrobial screening of new BisSchiff bases and their acetyl oxadiazole azetidinone derivatives from pyromellitic diimid. Int J Res Pharm \& Chem, 6, 1-8.

ANACONA, J. \& ACOSTA, F. 2006. Synthesis and antibacterial activity of cephradine metal complexes. Journal of Coordination Chemistry, 59, 621-627.

ANUSHA, K., KUMAR, Y. P., PRASAD, M. V. \& GOPINATH, C. A review on 2-Azetidinones. Journal of Global Trends in Pharmaceutical Sciences, 6, 2388-2402.

ARSHAD, I., SAEED, A., CHANNAR, P. A., SHEHZADI, S. A., AHMED, M. N. \& SIDDIQ, M. 2020. Bis-Schiff bases of 2, 2'-dibromobenzidine as efficient corrosion inhibitors for mild steel in acidic medium. RSC Advances, 10, 4499-4511.

AZIZ, D. M. \& AZEEZ, H. J. 2020. Synthesis of new ß-lactam-N(thiazol-2-yl) benzene sulfonamide hybrids: Their in vitro antimicrobial and in silico molecular docking studies. Journal of Molecular Structure, 1222, 128904.

BEG, M. \& ATHAR, F. 2020. Pharmacokinetic and molecular docking studies of Achyranthes aspera phytocompounds to exploring potential anti-tuberculosis activity. $J$ Bacteriol Mycol Open Access, 8, 18-27.

ESSA, H. H., KANDIL, F. \& FALAH, A. 2012. Synthesis and identification of Schiff bases and bilogical activity new study. Iraqi Journal of Science, 53, 230-240.

HALVE, A., DUBEY, R., BHADAURIA, D., BHASKAR, B. \& BHADAURIA, R. 2006. Synthesis, antimicrobial screening and structure-activity relationship of some novel 2hydroxy-5-(nitro-substituted phenylazo) benzylidine anilines. Indian journal of pharmaceutical sciences, 68.

HASSAN, S. A. 2019. Synthesis, Spectroscopic study and Biological activity of some New Heterocyclic compounds derived from Sulfadiazine. Zanco Journal of Pure and Applied Sciences, 31, 92-109.

HASSAN, S. A. \& AZIZ, D. M. 2021. Synthesis, in vitro Antimicrobial assay and Molecular Docking Studies of some new Symmetrical Bis-Schiff Bases and their 2-Azetidinones. Zanco Journal of Pure and Applied Sciences, 33, 34-50.

JARRAHPOUR, A., KHALILI, D., DE CLERCQ, E., SALMI, C. \& BRUNEL, J. M. 2007. Synthesis, antibacterial, antifungal and antiviral activity evaluation of some new bis-Schiff bases of isatin and their derivatives. Molecules, 12, 17201730 .

KAJAL, A., BALA, S., KAMBOJ, S., SHARMA, N. \& SAINI, V. 2013. Schiff bases: a versatile pharmacophore. Journal of Catalysts, 2013, 1-14.

KAUR, R., SINGH, R., AHLAWAT, P., KAUSHIK, P. \& SINGH, K. 2020a. Contemporary advances in therapeutic portfolio of 2-azetidinones. Chemical Biology Letters, 7, 13-26.

KAUR, R., SINGH, R., KUMAR, A., KAUR, S., PRIYADARSHI, N., SINGHAL, N. K. \& SINGH, K. 2020b. 1, 2, 3-Triazole $\beta$ lactam conjugates as antimicrobial agents. Heliyon, 6, e04241.

KAUR, R., TRIPATHI, D., SINGH, K. \& SINGH, R. 2018. Recent advances in $\beta$-lactam chemistry. Integrated Research Advances, 5, 52-57.

KHAN, K. M., KHAN, M., AMBREEN, N., RAHIM, F., MUHAMMAD, B., ALI, S., HAIDER, S. M., PERVEEN, S. \& CHOUDHARY, M. 2011. Bis-Schiff bases of isatins: a new class of antioxidant. J. Pharm. Res, 4, 3402-3404.

KHAN, T., YADAV, R. \& GOUND, S. S. 2018. An Efficient Synthesis and Antibacterial Activity of Some Novel 2-Azetidinone Derivatives of 4H-1, 2, 4-Triazoles Under Mild Conditions. Journal of Heterocyclic Chemistry, 55, 1042-1047.

LIANG, C., LIU, Z., LIANG, Q., HAN, G.-C., HAN, J., ZHANG, S. \& FENG, X.-Z. 2019. Synthesis of 2-aminofluorene bisSchiff base and corrosion inhibition performance for carbon steel in HCl. Journal of Molecular Liquids, 277, 330-340.

MCLOUGHLIN, E., MEEGAN, M. \& O'BOYLE, N. 2019. Stories from Staudinger: Synthesis of chiral beta-lactams, $5^{\text {th }}$ International Electronic Conference on Medicinal Chemistry.

MÉNDEZ, L., POEYLAUT-PALENA, A. A. \& MATA, E. G. 2018. Molecular diversity by olefin cross-metathesis on solid support. Generation of libraries of biologically promising $\beta$ lactam derivatives. Molecules, 23, 1193.

MOHATNTA, MISHRA, SAHU, MOHANTA \& BANERJEE. 2014. Insilico designing of Pyrazol-1-Y1 Azetidin-2-One derivatives as drug like molecules for possible inhibition of Anti Microbial 3GI9, 4AE5, 3FHU and 5COX target Proteins. International Journal of Drug Development and Research., 6, 78-91.

MY, N. H., HIRAO, H., VAN, D. U. \& MOROKUMA, K. 2011. Computational studies of bacterial resistance to $\beta$-lactam antibiotics: mechanism of covalent inhibition of the penicillin-binding protein 2a (PBP2a). Journal of chemical information and modeling, 51, 3226-3234.

N'DRI, J. S., KABLAN, A., OUATTARA, B., KONÉ, M., OUATTARA, L., KODJO, C. G. \& ZIAO, N. 2019. QSAR Studies of the Antifungal Activities of $\alpha$ Diaminophosphonates Derived from Dapsone by DFT Method. Journal of Materials Physics and Chemistry, 7, 17.

NAZERUDDIN, K. P. A. G. M. 2016. synthesis characterization and antimicrobial evaluation of some novel bis schiff bases. Journal of Chemical and Pharmaceutical Research, 8, 906911.

NWORIE, F. S. 2016. Bis (salicylidene) ethylenediamine (salen) and bis (salicylidene) ethylenediamine-metal complexes: From structure to biological activity. Journal of Analytical \& Pharmaceutical Research, 3, 76-85.

PAGADALA, R., MESHRAM, J. S., CHOPDE, H. N. \& JETTI, V. 2010. Synthesis of novel bis ( $\beta$-Lactams) from bis (ketene) and imines. Inter. J. Chem. Tech. Pharma, 2, 1581-1585.

PATEL HM, RAJANI DP, SHARMA MG, BHATT HG, 2019. Synthesis, molecular docking and biological evaluation of mannich products based on thiophene nucleus using ionic liquid. Journal of Letters in Drug Design, 16, 119-126.

PETRUS, M., BOUWER, R., LAFONT, U., ATHANASOPOULOS, S., GREENHAM, N. \& DINGEMANS, T. 2014. Smallmolecule azomethines: organic photovoltaics via Schiff 
base condensation chemistry. Journal of Materials Chemistry A, 2, 9474-9477.

SHARMA, M., SAHU, N., KOHLI, D., CHATURVEDI, S. \& SHARMA, S. 2009. Synthesis, Characterazation and biological activity of some 1-(Nicotinylamino)-2substituted Azetidine-4-ones as potential Antibacterial agents. Digest Journal of Nanomaterials \& Biostructures 4, 361-367.

SHINDE, A., ZANGADE, S., CHAVAN, S. \& TIWDE, S. 2015. Yeni bis-2-azetidinon ve bis-4-tiyazolidinon türevlerinin sentezi ve antioksidan aktivitesi. Journal of the Turkish Chemical Society Section A: Chemistry, 2, 22-31.

SHNEINE, J., AL-ARAJI, Y. \& SHAYEA, A. 2017. Synthesis of BisHeterocyclic Compounds and Study their Antimicrobial
Activity. International Journal of Science and Research, 6, 1387-1392.

SHOCKRAVI, A., SADEGHPOUR, M. \& OLYAEI, A. 2009. A convenient synthesis of novel symmetrical bis-Schiff bases of 2, 2'-thio-bis [4-methyl (2-aminophenoxy) phenyl ether] in solution and under solvent-free conditions. Journal of Chemical Research, 2009, 656-658.

TAHA, M., SAIN, A. A., ALI, M., RAHIM, F., ISMAIL, N. H., ADENAN, M. I., IMRAN, S., AL-HARRASI, A., NAWAZ, F. \& IQBAL, N. 2020. Synthesis of symmetrical bis-Schiff base disulfide hybrids as highly effective antileishmanial agents. Bioorganic chemistry, 99, 103819.

TROISI, L., GRANITO, C. \& PINDINELLI, E. 2010. Novel and recent synthesis and applications of $\beta$-lactams. Heterocyclic scaffolds I. Springer.

APPENDIX

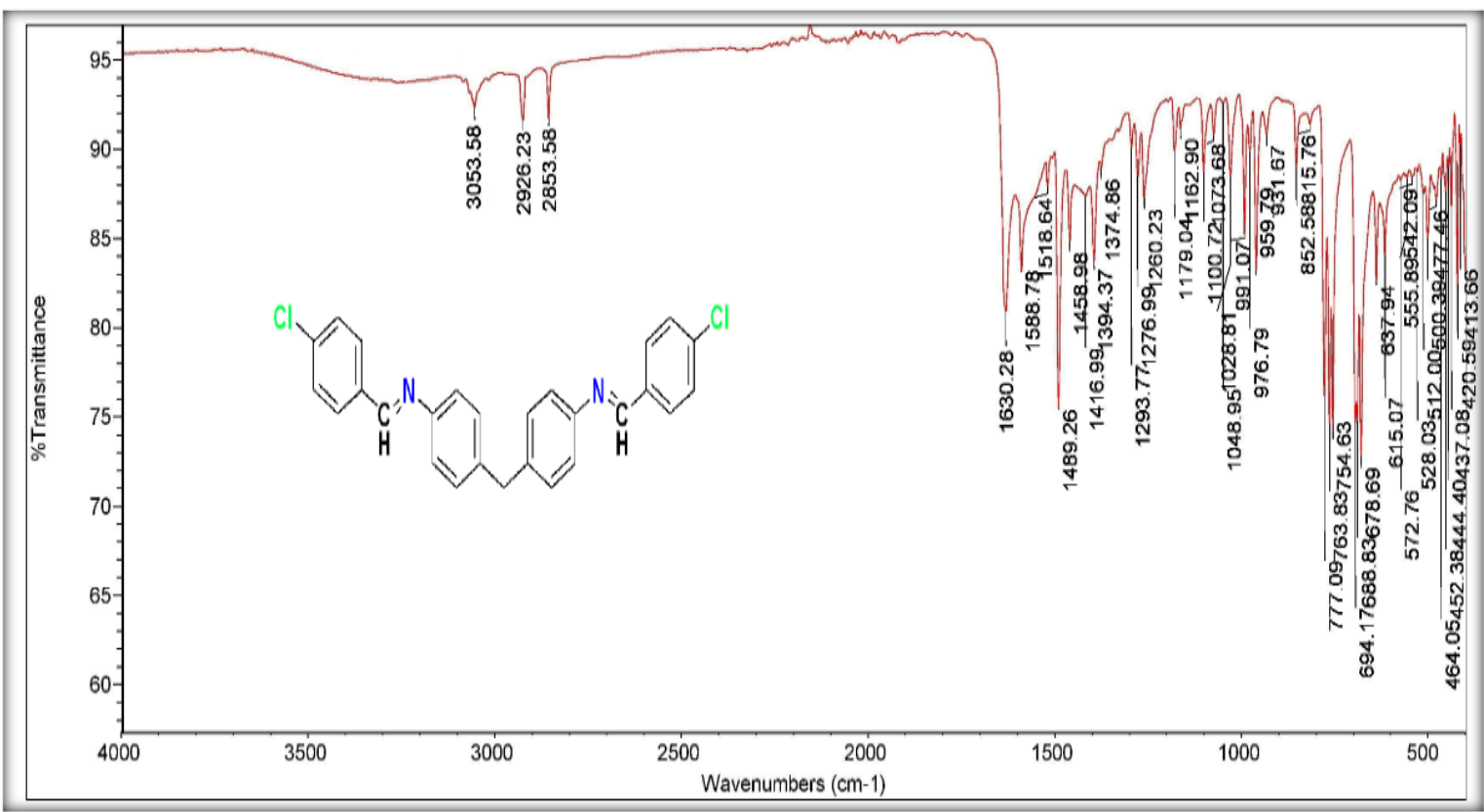

Figure 4. FT-IR Spectrum of $N, N^{\prime}$-(methylenebis(4,1-phenylene))bis(1-(4-chlorophenyl)methanimine) (3e)

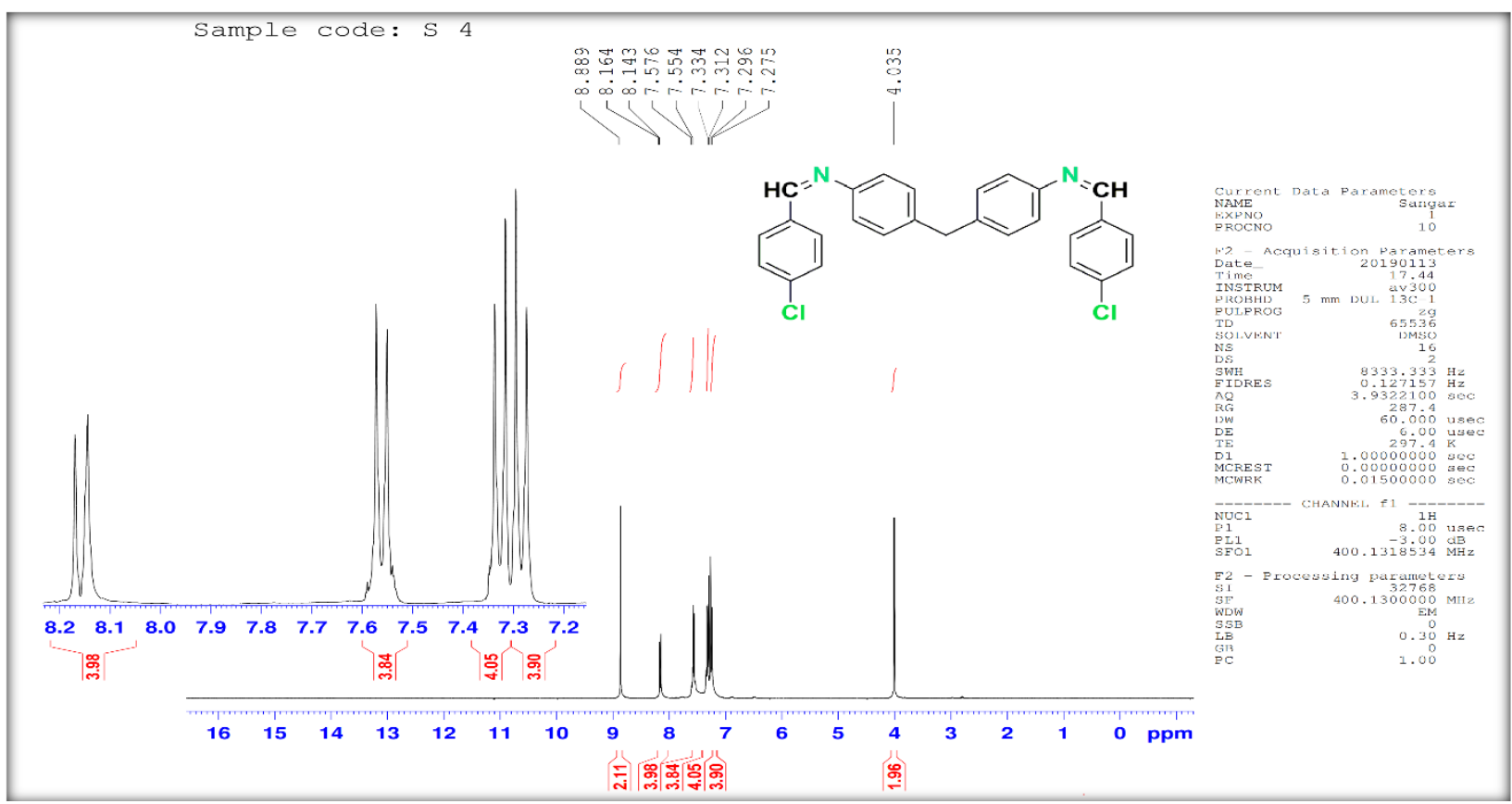

Figure 5. ${ }^{1} \mathrm{H}-\mathrm{NMR}$ Spectrum of $N, N^{\prime}$-(methylenebis(4,1-phenylene))bis(1-(4-chlorophenyl)methanimine) (3e) 


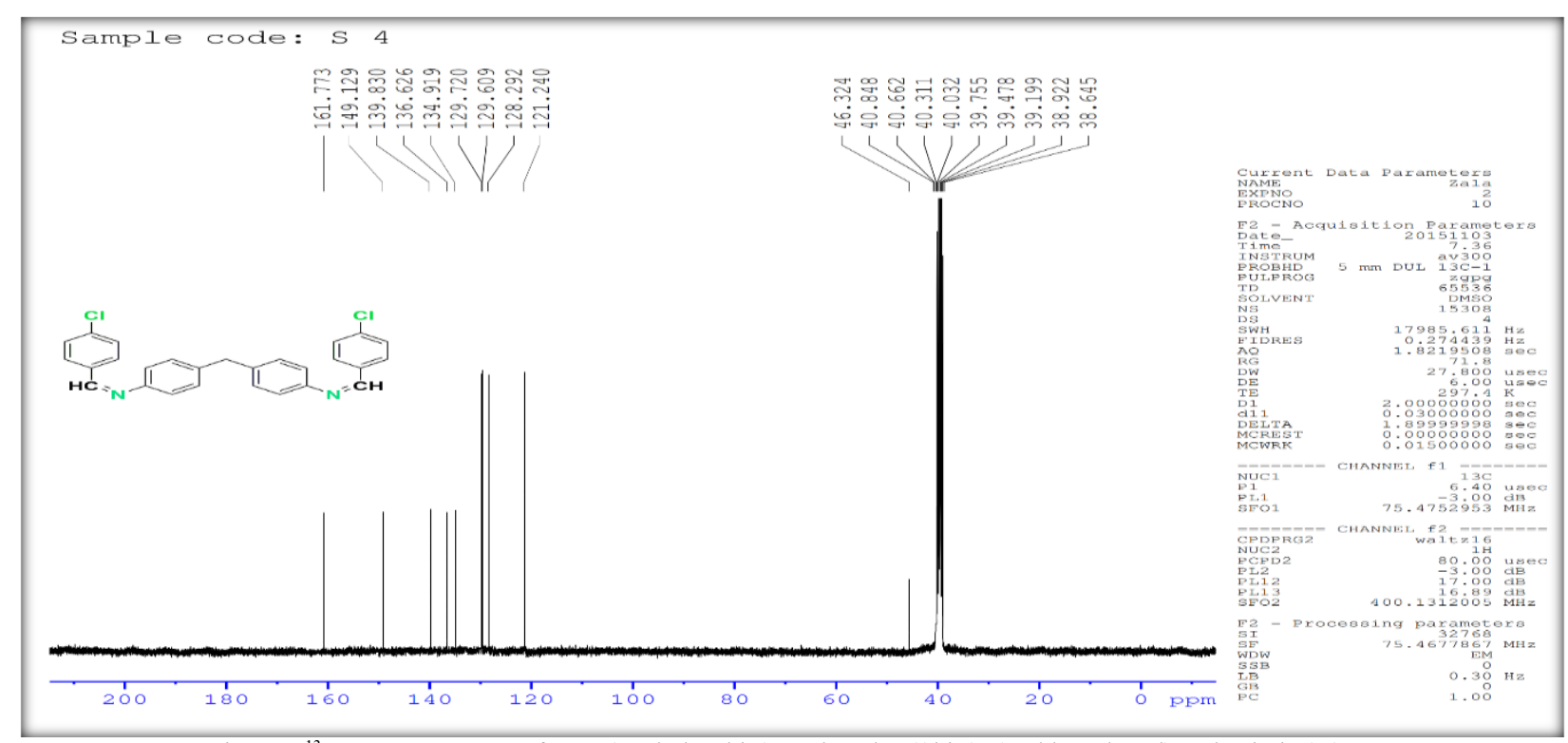

Figure $6:{ }^{13} \mathrm{C}$-NMR Spectrum of $N, N^{\prime}$-(methylenebis(4,1-phenylene))bis(1-(4-chlorophenyl)methanimine) (3e)

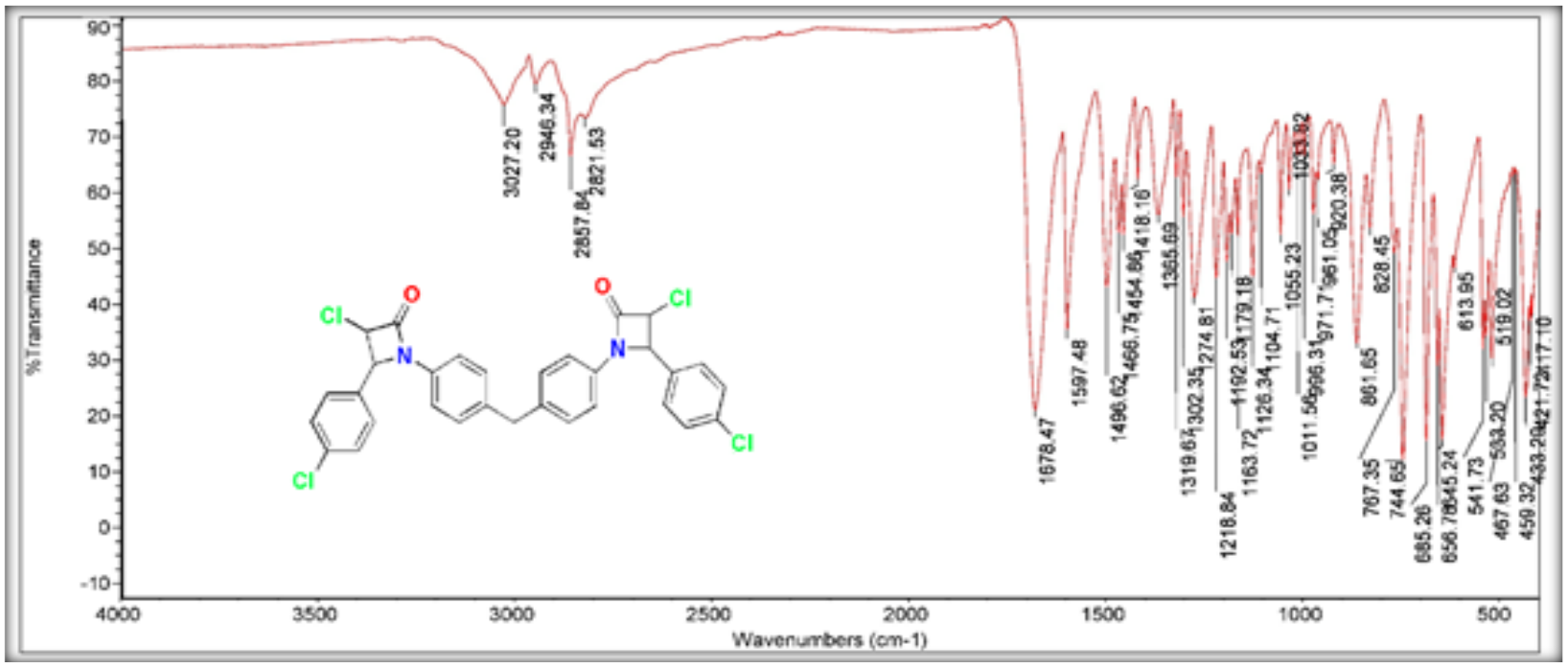

Figure 7. FT-IR Spectrum oftrans-1,1'-(methylenebis(4,1-phenylene))-trans-bis(3-chloro-4-(4-chlorophenyl) azetidin-2-one) (4e)

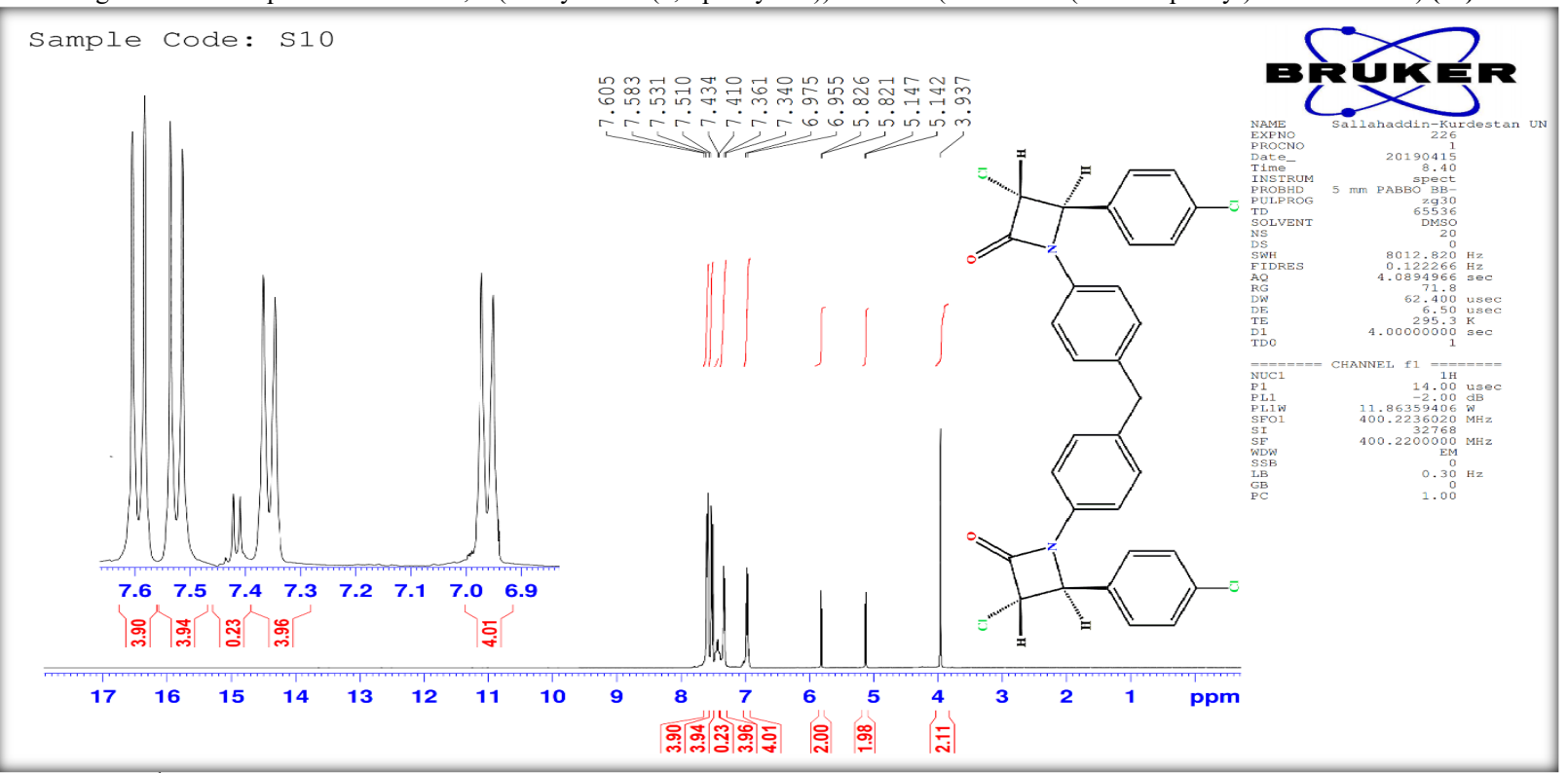

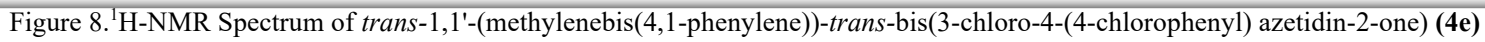




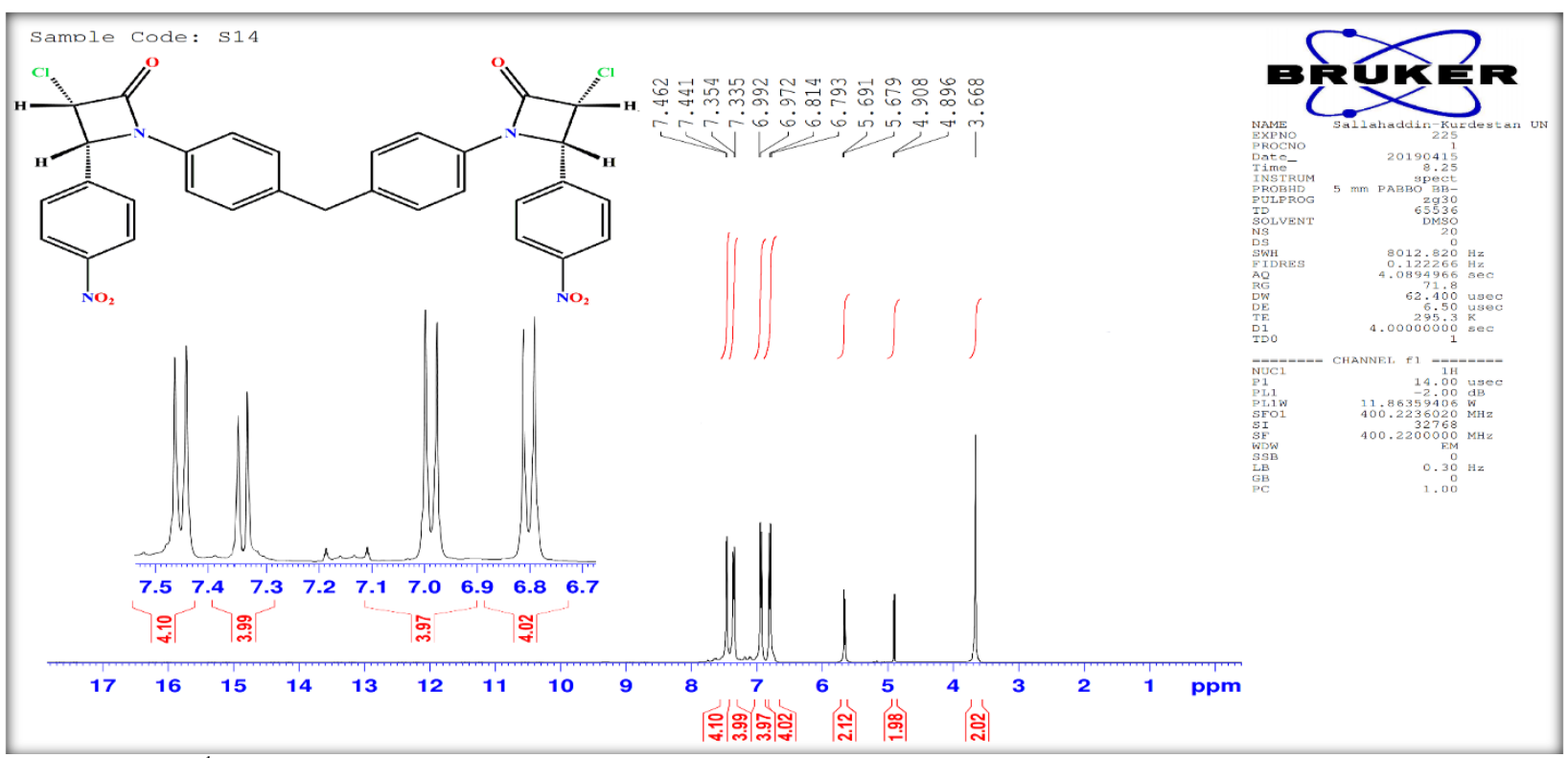

Figure 9. ${ }^{1}$ H-NMR Spectrum of $c i s-1,1^{1}$-(methylenebis(4,1-phenylene))-cis-bis(3-chloro-4-(4-nitrophenyl) azetidin-2-one) (4d)

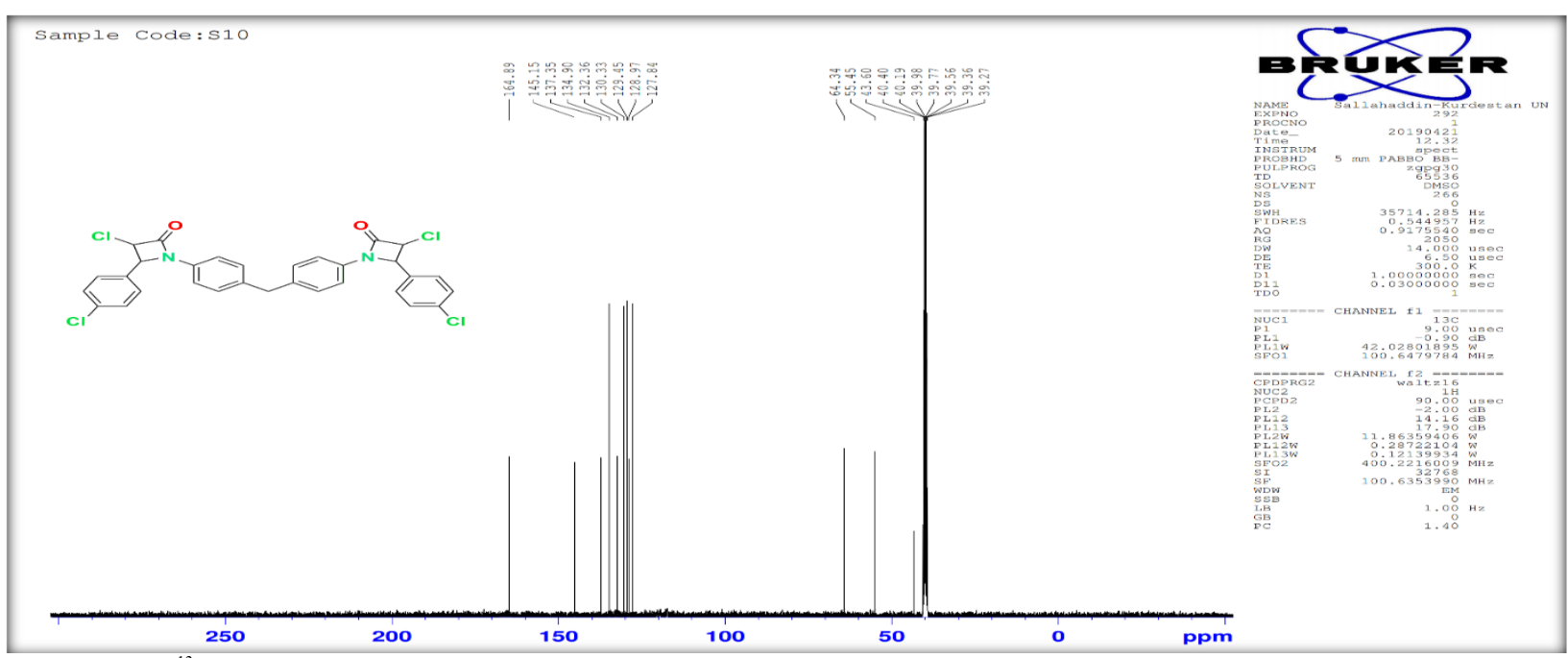

Figure $10 .{ }^{13} \mathrm{C}$-NMR Spectrum of trans-1,1'-(methylenebis(4,1-phenylene))-trans-bis(3-chloro-4-(4-chlorophenyl) azetidin-2-one) (4e) 